\title{
QCD Thermodynamics with Three Flavors of Improved Staggered Quarks
}

\author{
C. Bernard \\ Department of Physics, Washington University, St. Louis, MO 63130, USA \\ T. Burch \\ Institut für Theoretische Physik, Universität Regensburg, D-93040 Regensburg, Germany \\ C. DeTar and J. Osborn \\ Physics Department, University of Utah, Salt Lake City, UT 84112, USA \\ Steven Gottlieb \\ Department of Physics, Indiana University, Bloomington, IN 47405, USA \\ E.B. Gregory and D. Toussaint \\ Department of Physics, University of Arizona, Tucson, AZ 85721, USA \\ U.M. Heller \\ American Physical Society, One Research Road, Box 9000, Ridge, NY 11961-9000 \\ R. Sugar \\ Department of Physics, University of California, Santa Barbara, CA 93106, USA
}

(Dated: October 24, 2018) 


\begin{abstract}
We report on a study of QCD thermodynamics with three flavors of quarks, using a Symanzik improved gauge action and the Asqtad $O\left(a^{2}\right)$ improved staggered quark action. Simulations were carried out with lattice spacings $1 / 4 T, 1 / 6 T$ and $1 / 8 T$ both for three degenerate quarks with masses less than or equal to the strange quark mass, $m_{s}$, and for degenerate up and down quarks with masses in the range $0.1 m_{s} \leq m_{u, d} \leq 0.6 m_{s}$, and the strange quark mass fixed near its physical value. We present results for standard thermodynamics quantities, such as the Polyakov loop, the chiral order parameter and its susceptibility. For the quark masses studied to date we find a rapid crossover rather than a bona fide phase transition. We have carried out the first calculations of quark number susceptibilities with three flavors of sea quarks. These quantities are of physical interest because they are related to event-by-event fluctuations in heavy ion collision experiments. Comparison of susceptibilities at different lattice spacings show that our results are close to the continuum values.
\end{abstract}

PACS numbers: 12.38.Gc, 11.10.Wx, 12.38.Mh 


\section{INTRODUCTION}

Under ordinary laboratory conditions quarks and gluons, the fundamental constituents of quantum chromodynamics (QCD), are bound into hadrons. However, as the temperature or density is increased, the forces among quarks and gluons weaken, and one expects to find a phase transition or crossover to a new state of matter, a quark-gluon plasma. The plasma was a dominant state of matter in the early development of the universe, and a primary objective of current relativistic heavy ion collision experiments is to observe the plasma and determine its properties. The behavior of strongly interacting matter in the vicinity of the phase transition or crossover is inherently a strong coupling problem, which at present can only be addressed from first principles through lattice gauge theory calculations. Among the issues that can uniquely be addressed by lattice calculations are the nature of the transition and the temperature at which it occurs, the properties of the plasma, including strange quark content, and the equation of state [1].

In this paper we report on a study of the phase diagram of high temperature QCD with three flavors of quarks using improved gauge and quark actions 2]. Most lattice studies of high temperature QCD have included only the up and down quarks, but the inclusion of the strange quark is an important feature of our work [3]. From chiral models it is expected that with two flavors of quarks there is no phase transition at all for physical values of the up and down quark masses; however, a strange quark could induce a first order transition,

or move a second order critical point closer to physical quark masses [4]. Furthermore, the production of strangeness is expected to be an important signal for the plasma in heavy ion experiments.

We are considering two cases in our study: 1) all three quarks have the same mass $m_{q}$; and 2) the two lightest mass quarks have equal mass $m_{u, d}$, while the mass of the third quark is fixed at approximately that of the strange quark $m_{s}$. We refer to these cases as $N_{f}=3$ and $N_{f}=2+1$, respectively. For $N_{f}=3$ we have worked with quark masses in the range $0.2 m_{s} \leq m_{q} \leq m_{s}$, while for $N_{f}=2+1$ we have carried out simulations with $0.1 m_{s} \leq m_{u, d} \leq 0.6 m_{s}$. We have monitored standard thermodynamic quantities such as the plaquette, the Polyakov loop, the chiral order parameter and its susceptibility. For the quark masses we have studied to date, we find rapid crossovers, which sharpen as the quark mass is reduced, rather than a bona fide phase transition. This is in agreement with earlier 
work [1]. We have also measured the quark number susceptibilities, which provide excellent signals for the crossover, and which are directly related to event-by-event fluctuations in heavy ion collisions [5]. The data indicate that our results for these susceptibilities are quite close to their continuum values.

\section{THE SIMULATIONS}

Our simulations are carried out with a one-loop Symanzik improved gauge action [6] and the Asqtad quark action [7]. Both the gauge and quark actions have all lattice artifacts removed through order $a^{2}$ at tree level, where $a$ is the lattice spacing, and are tadpole improved. Thus, the leading order finite lattice spacing artifacts are of order $a^{2} \alpha_{s}$ and $a^{4}$. We and others have used this action over the last few years to perform a wide variety of zero-temperature calculations [8]. It has a number of features that make it particularly well suited for high temperature studies. The Asqtad action has considerably better dispersion relations for free quarks than the standard Kogut-Susskind and Wilson actions [9], which markedly decreases lattice artifacts above the transition. This is also true of the P4 action used by the Bielefeld group in its studies of high temperature QCD [10]. The improvement in dispersion relations is illustrated in Fig. 1, where we plot the energy, pressure and quark number susceptibility for free massless quarks as a function of lattice spacing. The Asqtad action exhibits excellent scaling properties in the lattice spacing 11], which accelerates the approach to the continuum limit. Finally, taste symmetry breaking is much smaller for the Asqtad action, than for the conventional Kogut-Susskind action. Our spectrum studies with the Asqtad action indicate that for lattices with eight to ten time slices, the kaon is heavier than the heaviest non-Goldstone pion in the neighborhood of the finite temperature transition or crossover, a condition which requires much smaller lattice spacings with the conventional Kogut-Susskind action. It is, of course, difficult to study the effects of the strange quark on the transition if this condition is not fulfilled.

We have used the refreshed hybrid molecular dynamics $\mathrm{R}$ algorithm [12] to generate gauge configurations. In integrating the molecular dynamics equations of motion we used a time step $d t$ equal to the smaller of 0.02 and $\frac{2}{3} m_{u, d}$. The momenta conjugate to the gauge fields were refreshed every molecular dynamics time unit, which consisted of $1 / d t$ time steps. In the vicinity of the crossover we generated 2,000 equilibrated molecular dynamics time units 
at each value of the gauge coupling and quark masses we studied. The runs well above and below the crossover were shorter. Measurements of standard thermodynamics quantities were made after each time unit, and gauge configurations were saved every five time units for separate measurements of the quark number and $\bar{\psi} \psi$ susceptibilities. Ten random sources were used in the measurements of $\bar{\psi} \psi$ made at the end of each time unit, and 100 random sources were used in the calculations of the susceptibilities. The values of the chiral order parameter obtained in these two measurements agreed to within statistics.

We have attempted to vary the temperature while keeping all other physical quantities constant. To this end, for the $N_{f}=3$ study we have performed a set of spectrum calculations at lattice spacings $a=0.125 \mathrm{fm}$ and $0.18 \mathrm{fm}$ with $m_{q}=m_{s}, 0.6 m_{s}, 0.4 m_{s}$, and $0.2 m_{s}$. We determine the lattice spacing from the static $\bar{Q} Q$ potential, and express it and other dimensionful quantities in terms of $r_{1}$ defined by $r_{1}^{2} F_{\bar{Q} Q}\left(r_{1}\right)=1$. Using results from the 1S-2S and 1P-1S splitting in the $\Upsilon$ spectrum [13], we have found $r_{1}=0.317 \pm 0.007 \mathrm{fm}$ in the continuum and chiral limits [14]. We determine $m_{s}$ from the requirement that $m_{\eta_{s s}} / m_{\phi} \approx$ 0.673 , where $\eta_{s s}$ is an "unmixed" pseudoscalar meson made of an $s$ and $\bar{s}$ quark. We would like to carry out our thermodynamics studies with three equal mass quarks for $m_{\eta_{s s}} / m_{\phi}$ fixed, but this quantity will, of course, vary slightly with lattice spacing if we keep $m_{q} / m_{s}$ fixed. So, at $a=0.18 \mathrm{fm}$ we perform linear interpolations of $m_{\eta_{s s}}^{2}$ and $m_{\phi}$ in the quark mass to determine the precise values of $m_{q}$ for which $m_{\eta_{s s}} / m_{\phi}$ will take on the values found at $a=0.125 \mathrm{fm}$. Then to determine the values of $a m_{q}, a$ and $T=1 / a N_{t}$ for thermodynamics studies with other values of $a$, we perform interpolations or extrapolations with a form due to Allton, inspired by asymptotic freedom [15]. We first define

$$
f\left(g^{2}\right)=\left(b_{0} g^{2}\right)^{-b_{1} /\left(2 b_{0}^{2}\right)} e^{-1 / 2 b_{0} g^{2}},
$$

where $b_{0}$ and $b_{1}$ are the universal beta-function coefficients for massless three-flavor QCD, and $g^{2}$ is the bare lattice coupling. $f\left(g^{2}\right)$ is basically $a / \Lambda_{L}$. We then determine $a$ as a function of $g^{2}$ from the interpolation formula

$$
a\left(g^{2}\right) / r_{1}=c_{0} f\left(g^{2}\right)\left[1+c_{2} g^{2} f^{2}\left(g^{2}\right)\right] .
$$

The second term in Eq. (21) is an $O\left(g^{2} a^{2}\right)$ correction to the asymptotic freedom formula. The coefficients $c_{0}$ and $c_{2}$ are determined from the measured values of $a / r_{1}$ at $a=0.18$ and 
$0.125 \mathrm{fm}$. A similar interpolation formula is used to determine the lattice quark mass along the line of fixed $m_{\eta_{s s}} / m_{\phi}$,

$$
a m_{q}\left(g^{2}\right)=d_{0}\left(b_{0} g^{2}\right)^{-4 / 9} f\left(g^{2}\right)\left[1+d_{2} g^{2} f^{2}\left(g^{2}\right)\right] .
$$

Here we have included the anomalous dimension of the mass. In the initial stages of our work we simply made linear interpolations of $\ln (a)$ and $\ln \left(a m_{q}\right)$ in $1 / g^{2}$ between the anchor points at $a=0.18$ and $0.125 \mathrm{fm}$, which gave results in good agreement with those of Eqs. (2) and (3). However, use of the more sophisticated formulae became critical for points outside the anchors.

Our approach for thermodynamics studies with $m_{u, d}<m_{s}$ is quite similar. In this case we wish to vary the temperature keeping both $m_{\pi} / m_{\rho}$ and $m_{\eta_{s s}} / m_{\phi}$ fixed. We have carried out spectrum studies at $a=0.125$ and $0.18 \mathrm{fm}$ for light quark masses $m_{u, d}=m_{s}, 0.6 m_{s}$, $0.4 m_{s}, 0.2 m_{s}$, and $0.1 m_{s}$, and at $a=0.09 \mathrm{fm}$ with $m_{\mu, d}=m_{s}, 0.4 m_{s} 0.2 m_{s}$ and $0.1 m_{s}$ all with the mass of the heavy quark fixed close to $m_{s}$ [9, 14]. The strange quark mass was determined from the spectrum calculations with three equal mass quarks. In our spectrum runs at $a=0.125 \mathrm{fm}[9]$ we found that $m_{\eta_{s s}}$ varied by less than $2 \%$ and $m_{\phi}$ by less than $1 \%$ for $0.2 m_{s} \leq m_{u, d} \leq m_{s}$, and the heavy quark mass held fixed at $m_{s}$. So, the neglect of the dependence of $m_{\eta_{s s}}$ and $m_{\phi}$ on $m_{u, d}$ is well justified. In these studies we performed linear interpolations of $m_{\pi}^{2}$ and $m_{\rho}$ at $a=0.18 \mathrm{fm}$ to determine the values of $m_{u, d}$ for which $m_{\pi} / m_{\rho}$ takes on the values found at $a=0.125 \mathrm{fm}$. Then, for other values of $a$ we interpolate or extrapolate $a / r_{1}$ using Eq. (2), and $a m_{u, d}$ and $a m_{s}$ using Eq. (3). We again determine the values of $c_{0}, c_{2}, d_{0}$ and $d_{2}$ from the spectrum runs at $a=0.125 \mathrm{fm}$ and $0.18 \mathrm{fm}$. For $m_{u, d}=m_{s}, 0.4 m_{s}, 0.2 m_{s}$ and $0.1 m_{s}$, where we have spectrum data at $a=0.09 \mathrm{fm}$, we can add $f^{4}\left(g^{2}\right)$ terms to the right hand sides of Eqs. (2) and (3). These added terms make only a few percent difference in the lattice spacing and quark masses extrapolated to $10 T_{C}$, giving us confidence in the interpolations and extrapolations used in our study. We have recently combined data from all zero temperature runs we have made to date to obtain a smooth interpolation formula of $\ln \left(r_{1} / a\right)$ as a function of quark mass and gauge coupling [14]. The results are in excellent agreement with those obtained from Eq. (2). 


\section{RESULTS FOR $\mathrm{N}_{\mathrm{f}}=3$}

For three equal mass quarks, $N_{f}=3$, we have carried out thermodynamics studies on lattices with four, six and eight time slices, and aspect ratio $N_{s} / N_{t}=2$. Here $N_{s}$ and $N_{t}$ are the spatial and temporal dimensions of the lattice in units of the lattice spacing. We also performed simulations with aspect ratio three for $N_{t}=4$, and obtained results that are indistinguishable from those with aspect ratio two. The spectrum calculations and interpolations described above allowed us to determine the values of the quark mass, $m_{q}$, that keep $m_{\eta_{s s}} / m_{\phi}$ fixed as the gauge coupling is varied. They also enabled us to determine the value of the lattice spacing, and therefore the temperature for each run.

In Fig. 2 we plot the real part of the Polyakov loop as a function of temperature on $16^{3} \times 8$ lattices. In this figure, as elsewhere, we give the values of $m_{q} / m_{s}$ for $a=0.125 \mathrm{fm}$. The corresponding values of $m_{\eta_{s s}} / m_{\phi}$ are given in Table [. The Polyakov loop shows a crossover from confined behavior at low temperature to deconfined behavior at high temperature. There is a slight trend for the temperature dependence of the Polyakov loop to be steeper for larger quark masses. This is to be expected, since at sufficiently large quark masses, it is a bona fide order parameter. The insensitivity of the Polyakov loop to the quark mass at fixed temperature or lattice spacing is, perhaps, not surprising. We have determined the lattice spacing from the heavy quark potential, and in our spectrum runs we adjusted the coupling constant to keep the lattice spacing fixed as the quark mass is varied. Since the Polyakov loop, like the heavy quark potential, is determined from measurements of purely gluonic operators, our procedure is likely to minimize the dependence of the Polyakov loop on the quark mass.

The number of conjugate gradient iterations varies as the temperature is changed reflecting a changing condition number for the Dirac operator as the spectrum of states changes. We show this quantity in Fig. 3 for the four values of the quark mass we have studied on $16^{3} \times 8$ lattices. The sharpening of the crossover as the quark mass is reduced is evident. The $\bar{\psi} \psi$ susceptibility provides a more physical signal for the crossover. It is given by

$$
\chi_{\text {tot }}=\frac{\partial}{\partial m}\langle\bar{\psi} \psi\rangle=\frac{T}{V_{s}}\left\langle\operatorname{Tr} M^{-1} \operatorname{Tr} M^{-1}\right\rangle-\frac{T}{V_{s}}\left\langle\operatorname{Tr} M^{-2}\right\rangle-\frac{T}{V_{s}}\left\langle\operatorname{Tr} M^{-1}\right\rangle^{2},
$$

where $M$ is the fermion matrix, $T$ the temperature, and $V_{s}$ the spatial volume. The traces 


\begin{tabular}{|c|c|c|c|}
\hline \multicolumn{2}{|c|}{$N_{f}=3$} & \multicolumn{2}{c|}{$N_{f}=2+1$} \\
\hline \hline$m_{q} / m_{s}$ & $m_{\eta_{s s}} / m_{\phi}$ & $m_{u, d} / m_{s}$ & $m_{\pi} / m_{\rho}$ \\
\hline 1.0 & 0.673 & 1.0 & 0.673 \\
0.6 & 0.583 & 0.6 & 0.582 \\
0.4 & 0.504 & 0.4 & 0.509 \\
0.2 & 0.404 & 0.2 & 0.392 \\
& & 0.1 & 0.298 \\
\hline
\end{tabular}

TABLE I: In the first column we show the value of $m_{q} / m_{s}$ at lattice spacing $0.125 \mathrm{fm}$, which produced the $m_{\eta_{s s}} / m_{\phi}$ ratio shown in the second column for spectrum calculations with three equal mass quarks. In the third column we give the value of $m_{u, d} / m_{s}$ which produced the $m_{\pi} / m_{\rho}$ ratio shown in the fourth column for spectrum calculations with two equal mass light quarks, and the mass of the heavy quark fixed at $m_{s}$.

in Eq. (41) were, as usual, evaluated using the identity

$$
\operatorname{Tr} O=\left\langle R^{*} O R\right\rangle_{R}
$$

where \langle\rangle$_{R}$ indicates an average over vectors, $R$, of Gaussian random numbers. We used 100 vectors of random numbers for each gauge configuration. Of course, care must be taken not to use the same vector of random numbers in evaluating the product of traces in the first term of Eq. (4). We plot $\chi_{\text {tot }}$ in Fig. 4 for quark masses $m_{s}, 0.6 m_{s}, 0.4 m_{s}$, and $0.2 m_{s}$ on $12^{3} \times 6$ lattices. Note the increase in the height of the peak as the quark mass is decreased.

In Figs. 5 and $[6$ we show the chiral order parameter, $\bar{\psi} \psi$ as a function of temperature for $m_{q}=m_{s}, 0.6 m_{s}, 0.4 m_{s}$, and $0.2 m_{s}$ on $12^{3} \times 6$ and $16^{3} \times 8$ lattices, respectively. The bursts in these figures are linear extrapolations of $\bar{\psi} \psi$ in the quark mass to $m_{q}=0$ for fixed temperature. These figures suggest that for $m_{q}=0$ there is unlikely to be a phase transition for temperatures above $190 \mathrm{MeV}$, but one could occur at or below that value. For sufficiently high temperatures and small quark masses, one expects a linear extrapolation in the quark mass to be valid, so the vanishing of $\bar{\psi} \psi$ at $m_{q}=0$ indicates that the system is in the chiral symmetric phase. However, a non-vanishing value of the extrapolated $\bar{\psi} \psi$ could indicate a first order transition at a non-zero quark mass, or simply a breakdown in the validity of the 
linear extrapolation in the quark mass.

\section{RESULTS FOR $N_{f}=2+1$}

The $N_{f}=2+1$ thermodynamics studies were carried out primarily on $8^{3} \times 4,12^{3} \times 6$ and $16^{3} \times 8$ lattices. In addition, a number of runs were made on $18^{3} \times 6$ lattices to check for finite spatial size effects, which turned out to be negligible. In this phase of our work, we performed simulations with two degenerate light quarks and the heavy quark mass held approximately equal to that of the strange quark. The spectrum calculations and interpolations described above allowed us to determine curves of constant physics in the coupling constant-quark mass plane, and to determine the lattice spacing, and therefore the temperature for each run.

In Fig. [7 we plot the real part of the Polyakov loop as a function of temperature on $16^{3} \times 8$ lattices for the four values of $m_{u, d}$ studied to date. As in the $N_{f}=3$ study, we observe a crossover from confined to deconfined behavior, rather than a sharp transition, and little dependence on the light quark mass. In Fig. 8 we show the number of conjugate gradient iterations needed for inversion of the Dirac operator for the light quarks on $16^{3} \times 8$ lattices, and in Fig. 9 we plot the $\bar{\psi} \psi$ susceptibility for the up and down quarks on $12^{3} \times 6$ lattices. In both figures the sharpening of the crossover as the light quark masses are reduced is evident.

In Figs. 10 and 11] we plot the chiral order parameter as a function of temperature on $12^{3} \times 6$ and $16^{3} \times 8$ lattices. The octagons, diamonds, squares and fancy crosses are data for $m_{u, d}=0.6 m_{s}, 0.4 m_{s}, 0.2 m_{s}$ and $0.1 m_{s}$ respectively, and the bursts are linear extrapolations in $m_{u, d}$ for fixed temperatures using the $0.2 m_{s}$ and $0.1 m_{s}$ points. The fact that the linear extrapolation of the chiral order parameter becomes slightly negative suggests that in this

region of temperature and quark mass $\bar{\psi} \psi$ is nonlinear, as would be expected if there were a bona fide critical point for $m_{u, d} \geq 0$.

\section{QUARK NUMBER SUSCEPTIBILITIES}

In order to study the quark number susceptibilities [16, 17, 18, 19, 20, 21], we introduce chemical potentials $\mu_{\alpha}$ coupled to a set of mutually commuting conserved charges $Q_{\alpha}$. The 
partition function can then be written

$$
Z=\exp \left[-\beta\left(H-\sum_{\alpha} \mu_{\alpha} Q_{\alpha}\right)\right] .
$$

The quark number susceptibilities can be related to event-by-event fluctuations in heavy ion collisions [5] by the fluctuation-dissipation theorem

$$
\chi_{\alpha, \beta}(T)=\left\langle\left(Q_{\alpha}-\left\langle Q_{\alpha}\right\rangle\right)\left(Q_{\beta}-\left\langle Q_{\beta}\right\rangle\right)\right\rangle \propto \frac{T}{V_{s}} \frac{\partial^{2} \log Z}{\partial \mu_{\alpha} \partial \mu_{\beta}},
$$

We work at $\mu_{\alpha}=0$, so the brackets, \langle\rangle , in Eq. (7) indicate averages weighted by the standard, real Euclidean action for $\mathrm{QCD}$, and $\left\langle Q_{\alpha}\right\rangle=0$. The charges we consider are bilinears in the quark fields, so it is possible to write $\chi_{\alpha, \beta}$ in the form

$$
\chi_{\alpha, \beta}=C_{\alpha, \beta}+D_{\alpha, \beta}
$$

where $C_{\alpha, \beta}$ is the contribution to $\chi_{\alpha, \beta}$ of connected diagrams in which quark fields from charge $Q_{\alpha}$ contract with those from $Q_{\beta}$, whereas $D_{\alpha, \beta}$ is the contribution of the disconnected diagrams in which quark fields within each charge contract.

If we take the charges to be the number operators for up, down and strange quarks, then because we have set $m_{u}=m_{d}, \chi$ takes the form

$$
\chi=\left(\begin{array}{ccc}
C_{l, l}+D_{l, l} & D_{l, l} & D_{l, s} \\
D_{l, l} & C_{l, l}+D_{l, l} & D_{l, s} \\
D_{l, s} & D_{l, s} & C_{s, s}+D_{s, s}
\end{array}\right)
$$

where the rows and columns of the matrix are labeled by $u, d$ and $s$ in that order, and $l$ stands for the equal up and down quark matrix elements.

It appears more physical to take the three independent charges to be the z-component of isospin, $Q_{I}$, the hypercharge, $Q_{Y}$, and the baryon number, $Q_{B}$, where in the continuum

$$
\begin{aligned}
Q_{I} & =\frac{1}{2} \int d^{3} x \Psi^{\dagger}(x) \lambda_{3} \Psi(x) \\
Q_{Y} & =\frac{1}{\sqrt{3}} \int d^{3} x \Psi^{\dagger}(x) \lambda_{8} \Psi(x) \\
Q_{B} & =\frac{1}{3} \int d^{3} x \Psi^{\dagger}(x) \cdot \Psi(x) .
\end{aligned}
$$


Here $\Psi(x)$ is a three-component column vector, whose components are the up, down and strange quark fields, and $\lambda_{3}$ and $\lambda_{8}$ are the standard diagonal generators of $\mathrm{SU}(3)$ in the fundamental representation, $\lambda_{3}=\operatorname{diag}(1,-1,0)$ and $\lambda_{8}=\operatorname{diag}(1,1,-2) / \sqrt{3}$. Using these charges, $\chi$ can be written in the form

$\chi=\left(\begin{array}{ccc}\frac{1}{2} C_{l, l} & 0 & 0 \\ 0 & \frac{2}{9}\left(C_{l, l}+2 C_{s, s}\right)+\frac{4}{9}\left(D_{l, l}-2 D_{l, s}+D_{s, s}\right) & \frac{2}{9}\left(C_{l, l}-C_{s, s}+2 D_{l, l}-D_{l, s}-D_{s, s}\right) \\ 0 & \frac{2}{9}\left(C_{l, l}-C_{s, s}+2 D_{l, l}-D_{l, s}-D_{s, s}\right) & \frac{1}{9}\left(2 C_{l, l}+C_{s, s}+4 D_{l, l}+4 D_{l, s}+D_{s, s}\right)\end{array}\right)$,

where the rows and columns are now labeled by $I, Y$ and $B$ in that order [22]. Note that in the $N_{f}=3$ simulations for which $m_{u}=m_{d}=m_{s}, C_{l, l}=C_{l, s}=C_{s, s}$ and $D_{l, l}=$ $D_{l, s}=D_{s, s}$, so $\chi$ is a diagonal matrix in this representation. There are then no correlations between fluctuations $I, Y$ and $B$. In the $N_{f}=2+1$ case the only correlations are between hypercharge and baryon number. Of course for $m_{u} \neq m_{d}$ there would be correlations among the fluctuations in all three charges. For temperatures below the phase transition or crossover, the lightest particle that can be excited by a chemical potential coupled to the z-component of isospin is the pion, while for hypercharge and baryon number chemical potentials it is the kaon and the nucleon, respectively. Above the transition temperature each of the chemical potentials can excite quark states that are much lighter than hadrons, so we expect the diagonal elements of $\chi$ to increase sharply in the vicinity of the transition, and they do.

As in the case of $\chi_{\text {tot }}$, these susceptibilities can be written as expectation values of traces of the quark matrices and their derivatives with respect to the chemical potential, and unbiased estimators of the traces can be expressed in terms of vectors of Gaussian random numbers [16]. Here too, we use 100 random vectors for each gauge configuration.

In Fig. 1 we plot the quark number susceptibility of massless free quarks for the standard Kogut-Susskind, Wilson, P4 and Asqtad actions. (With only one flavor of quark, there is, of course, only one susceptibility). One sees that as in the case of the energy and pressure, the susceptibility of the Asqtad action is significantly closer to the continuum result for small values of $N_{t}$ (large values of the lattice spacing) than that of the Kogut-Susskind action. In Fig. 12 we show $\chi_{I, I}$ for the four quark masses we have studied on $12^{3} \times 6$ lattices with $N_{f}=3$. One again sees the steepening of the crossover and its shift to lower temperature 
as the quark mass is decreased. In Fig. 13 we illustrate the dependence of $\chi_{I, I}$ on lattice spacing by plotting results at fixed quark mass for three values of $N_{t}$. The solid lines on the right of this figure indicate the values of the susceptibility for free quarks in the continuum and on the finite lattices on which the simulations were carried out.

In Fig. 14 we plot $\chi_{I, I}$ on $12^{3} \times 6$ lattices for $N_{f}=2+1$. As indicated in Eq. (13), this quantity is proportional to the light quark connected diagram, $C_{l, l}$. The disconnected diagrams are, of course noisier. As an example, we plot the light quark disconnected graph in Fig. 15. This quantity is given by

$$
D_{l, l}=\left\langle(B+Y / 2)^{2}\right\rangle-\langle(B+Y / 2)\rangle^{2}-\chi_{I, I} .
$$

We see in Fig. 15 that $D_{l, l}$ can be cleanly evaluated in the neighborhood of the crossover, the only region in which it is appreciable. Below the crossover one expects $\chi_{I, I}$ to be larger than $\chi_{B+Y / 2, B+Y / 2}$, since in this regime the lowest energy state that can be excited by a chemical potential coupled to $I_{z}$ is a pion, while the lowest energy state that can be excited by a chemical potential coupled to $B+Y / 2$ is a kaon. Our results suggest that vestiges of hadronic physics persist in the plasma at least up to $240 \mathrm{MeV}$. In Figs. [16] and [17 we show results for $\chi_{I, I}$ and for the strange quark number susceptibility, $C_{s, s}+D_{s, s}$, on lattices with 4, 6 and 8 time slices for a range of spatial volumes. There is no observable dependence on the spatial volume. The close agreement between the $N_{t}=6$ and 8 results here and for $N_{f}=3$ illustrates the excellent scaling properties of the action, and indicates that our results are close to the continuum ones. Finally, in Fig. 18 we plot the diagonal elements of the susceptibility matrix, $\chi_{I, I}, \chi_{Y, Y}$ and $\chi_{B, B}$, as a function of temperature for two light quarks with mass $0.2 m_{s}$ and one heavy quark with mass $m_{s}$ on $12^{3} \times 6$ lattices. $\chi_{Y, Y}$ and $\chi_{B, B}$ have been multiplied by factors of $3 / 4$ and $3 / 2$ respectively, so that the quantities plotted approach the same high temperature limit as $\chi_{I, I}$. Also shown is $\chi_{Y, B}$, the only non-zero off-diagonal matrix element of $\chi$ for $m_{u}=m_{d}$. It measures correlations between fluctuations in the hypercharge and baryon number. The coefficient of $\chi_{I, B}$ in this figure is the geometric mean of those for $\chi_{Y, Y}$ and $\chi_{B, B}$. 


\section{CONCLUSION}

For the quark masses we have studied to date, we find a rapid crossover, rather than a bona fide phase transition, for both $N_{f}=3$ and $N_{f}=2+1$. Our result for $N_{f}=3$ is consistent with recent work of Karsch et al. 23]. They find a first order phase transition for $N_{f}=3$ only for pion masses below 290(20) MeV for the standard Kogut-Susskind action and below 67(18) $\mathrm{MeV}$ for the improved P4 action on lattices with four time slices. The lightest quark mass used in [23] corresponded to a pion mass of $170 \mathrm{MeV}$, and reweighting techniques were used to extrapolate to lighter quark masses. Our lightest $N_{f}=3$ quark mass corresponds to a pion mass of approximately $340 \mathrm{MeV}$. Because of the large difference between standard and improved actions on these lattices, it seems particularly important to push our work at $N_{t}=6$ and 8 to smaller quark masses, and we intend to do so.

The small quark mass that seems to be required for a first order transition at $N_{f}=3$ strongly suggests that in the real world, $N_{f}=2+1$, there is no phase transition at the physical quark masses. With this assumption, we have estimated the critical temperature for $N_{f}=2+1$ at $m_{u, d}=0$ through an extrapolation of the form

$$
r_{1} T_{c}=c_{0}+c_{1}\left(m_{\pi} / m_{\rho}\right)^{d}+c_{2}\left(a T_{c}\right)^{2}
$$

where we evaluated $T_{c}$ for each value of $N_{t}$ and $m_{\pi} / m_{\rho}$ for which we have made measurements from the peak in the $\bar{\psi} \psi$ susceptibility. For a second order phase transition in the $\mathrm{O}(4)$ universality class at $m_{u, d}=0, d=2 / \beta \delta \approx 1.08$. We find that $T_{c}=169(12)(4) \mathrm{MeV}$ with a $\chi^{2}$ of 2.1 for 11 degrees of freedom. The first error is the fit error, the second from the uncertainty in $r_{1}$, taken as $0.317(7) \mathrm{fm}[14]$. To test the sensitivity of $T_{c}$ to $d$, we have also performed a fit with $d=2$, which yields $T_{c}=174(11)(4) \mathrm{MeV}$ with a $\chi^{2}$ of 1.5 for 11 degrees of freedom. So, the goodness of the fit does not allow us to prefer either of them.

Finally, we note that the agreement between the quark number susceptibilities on $N_{t}=6$ and 8 lattices is very encouraging, as it indicates that our results for these quantities are close to their continuum values. 


\section{ACKNOWLEDGMENTS}

Computations for this work were performed at Florida State University, Fermi National Accelerator Laboratory (FNAL), the National Center for Supercomputer Applications (NCSA), the National Energy Resources Supercomputer Center (NERSC), and the University of Utah (CHPC). This work was supported by the U.S. Department of Energy under contracts DE-FG02-91ER-40628, DE-FG02-91ER-40661, and DE-FG03-95ER-40906 and National Science Foundation grants PHY01-39929 and PHY00-98395. TB acknowledges current support from BMBF and GSI.

[1] For recent reviews of lattice studies of high temperature QCD see F. Karsch, Lect. Notes Phys. 583, 209 (2002); Nucl. Phys. A698, 199 (2002); S. Gottlieb, J. Phys. G30, S421 (2004).

[2] Preliminary results were reported in The MILC Collaboration: C. Bernard et al., Nucl. Phys. A702, 140 (2002); Nucl. Phys. B (Proc. Suppl.) 106, 429 (2002); 119, 523, (2003); and 129\& 130, 626 (2004); arXiv:hep-lat/0409097, to be published in Nucl. Phys. B (Proc. Suppl.)

[3] For earlier studies of three-flavor QCD see F. Brown et al., Phys. Rev. Lett. 65, 2491 (1990); Y. Iwasaki et al., Phys. Rev. D54, 7010 (1996); F. Karsch, E. Laermann, A. Peikert, and B. Sturm, Nucl. Phys. B (Proc.Suppl.) 73, 468 (1999); JLQCD Collaboration, S. Aoki et al., Nucl. Phys. (Proc. Suppl.) 73, 459 (1999).

[4] R.D. Pisarski and F. Wilczek, Phys. Rev. D 29, 338 (1984); S. Gavin, A.!Gocksch, and R.D. Pisarski, 49, 3079 (1994).

[5] B. Müller, Nucl. Phys. A 702, 281 (2002); V. Koch, M. Bleicher and S. Jeon, Nucl. Phys. A 702, 291 (2002); R.V. Gavai, Nucl. Phys. A 702, 299 (2002).

[6] K. Symanzik, in "Recent Developments in Gauge Theories", eds. G. 't Hooft et al., 313 (Plenum, New York, 1980).

[7] K. Orginos and D. Toussaint, Phys. Rev. D59, 014501 (1999) and Nuc. Phys. B (Proc. Suppl.) 99, 909 (199); G.P. Lepage, Phys. Rev. D 59, 074501 (1999); K. Orginos, D. Toussaint and R.L. Sugar, Phys. Rev. D 60, 054503 (1999).

[8] For a review of zero-temperature results obtained with the Asqtad action see S. Gottlieb, hep-lat/0310041, to be published in Nuc. Phys. B (Proc. Suppl.), and C.T.H. Davies et al., 
Phys. Rev. Lett. 92, 022001 (2004).

[9] The MILC Collaboration, C. Bernard et al., Phys. Rev. D 64, 054506 (2001).

[10] J.Engels, R. Joswig, F. Karsch, E. Laermann, M. Lutgemeier, and B. Petersson, Phys. Rev. B396, 210 (1997).

[11] The MILC Collaboration, C. Bernard, et al., Phys. Rev. D 61, 111502 (2000).

[12] S. Gottlieb, et al., Phys. Rev. D 35, 2531 (1987).

[13] M. Wingate, C.T.H. Davies, A. Gray, G.P. Lepage, and J. Shigemitsu, Phys.Rev.Lett. 92 (2004) 162001.

[14] C. Bernard et al., arXiv:hep-lat/0402030, submitted to Phys. Rev. D.

[15] C. Allton, Nucl. Phys. B (Proc. Suppl.) 53, 867 (1997); arXiv:hep-lat/9610016 (unpublished).

[16] S. Gottlieb et al., Phys. Rev. Lett. 59, 2247 (1987).

[17] R.V. Gavai and S. Gupta, Phys. Rev. D 64, 074506 (2001); Phys. Rev. D 65, 094515, (2002);

R.V. Gavai, S. Gupta and P. Majumdar, Phys. Rev. D 65, 054506 (2002).

[18] S. Ejiri et al., arXiv:hep-lat/0312006.

[19] J.P. Blaizot, E. Iancu and A. Rebhan, Phys. Lett. B523, 143 (2001), and Eur. Phys. J. C27, 433 (2003); A. Rebhan, arXiv:hep-ph/0301130.

[20] P. Chakrabatrty, M.G. Mustafa and M.H. Thoma, Eur. Phys. J. C23, 591 (2002), and Phys. Rev. D 68, 085012 (2003).

[21] A. Vuorinen, Phys. Rev. D 67, 074032 (2003), and Phys. Rev. D 68, 054017 (2003).

[22] Our normalization of the quark number susceptibilities differs from that used in studies of two-flavor QCD and in our earlier reports on this work. In our present notation $\chi_{I, I}=\chi_{N S} / 4$ and $\chi_{B+\frac{1}{2} Y, B+\frac{1}{2} Y}=\chi_{S} / 4$, where $\chi_{N S}$ and $\chi_{S}$ are commonly referred to as the non-singlet and singlet susceptibilities.

[23] F. Karsch et al., Nucl. Phys. B (Proc. Suppl.), 129 \& 130, 614 (2004). 

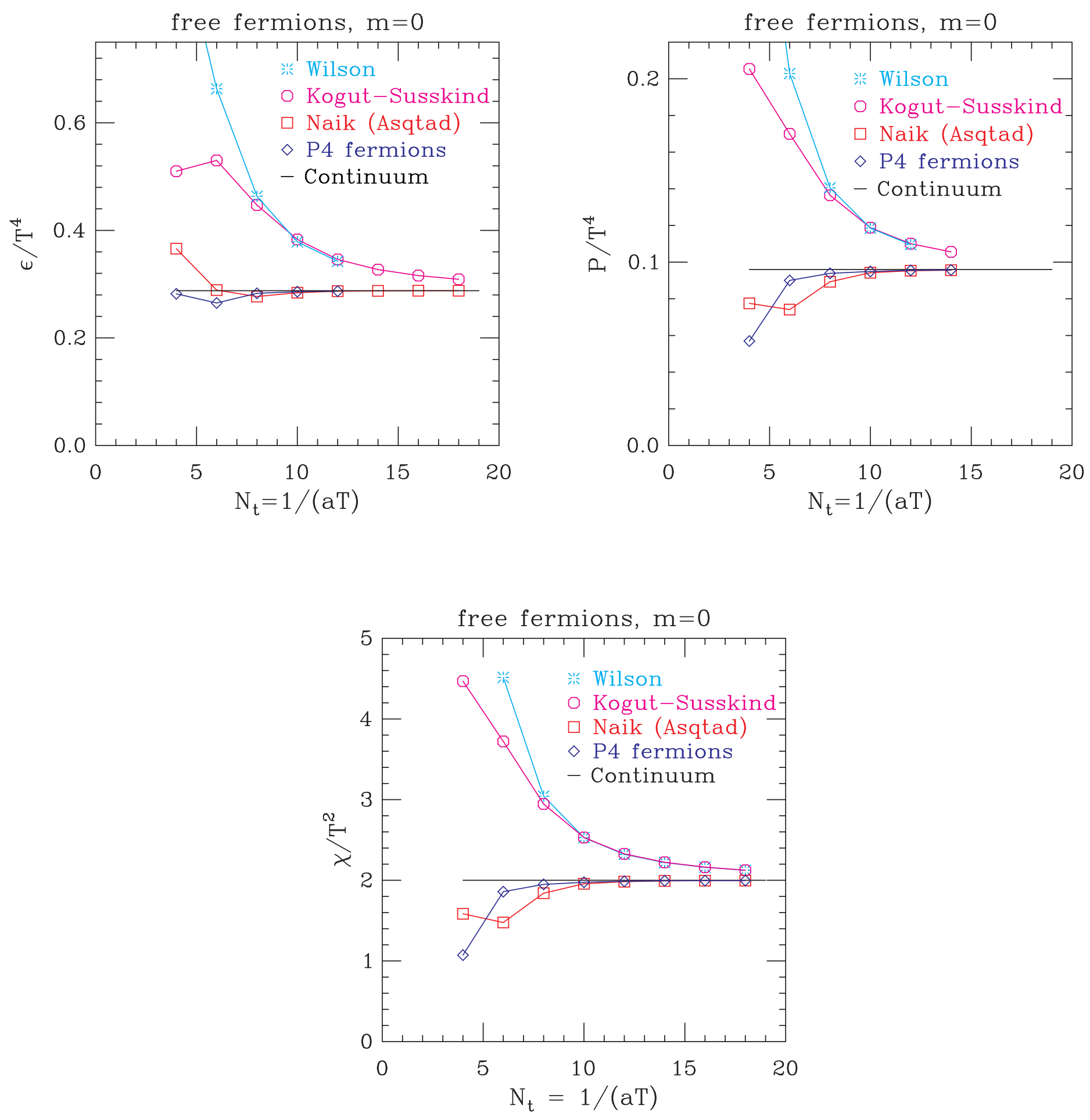

FIG. 1: The energy density, pressure and quark number susceptibility for free, massless quarks as a function of the temporal lattice size, $N_{t}$, for conventional and improved actions. For free fermions the Asqtad action reduces to the Naik action. The $P 4$ action is the improved staggered fermion action studied by the Bielefeld group [10]. 


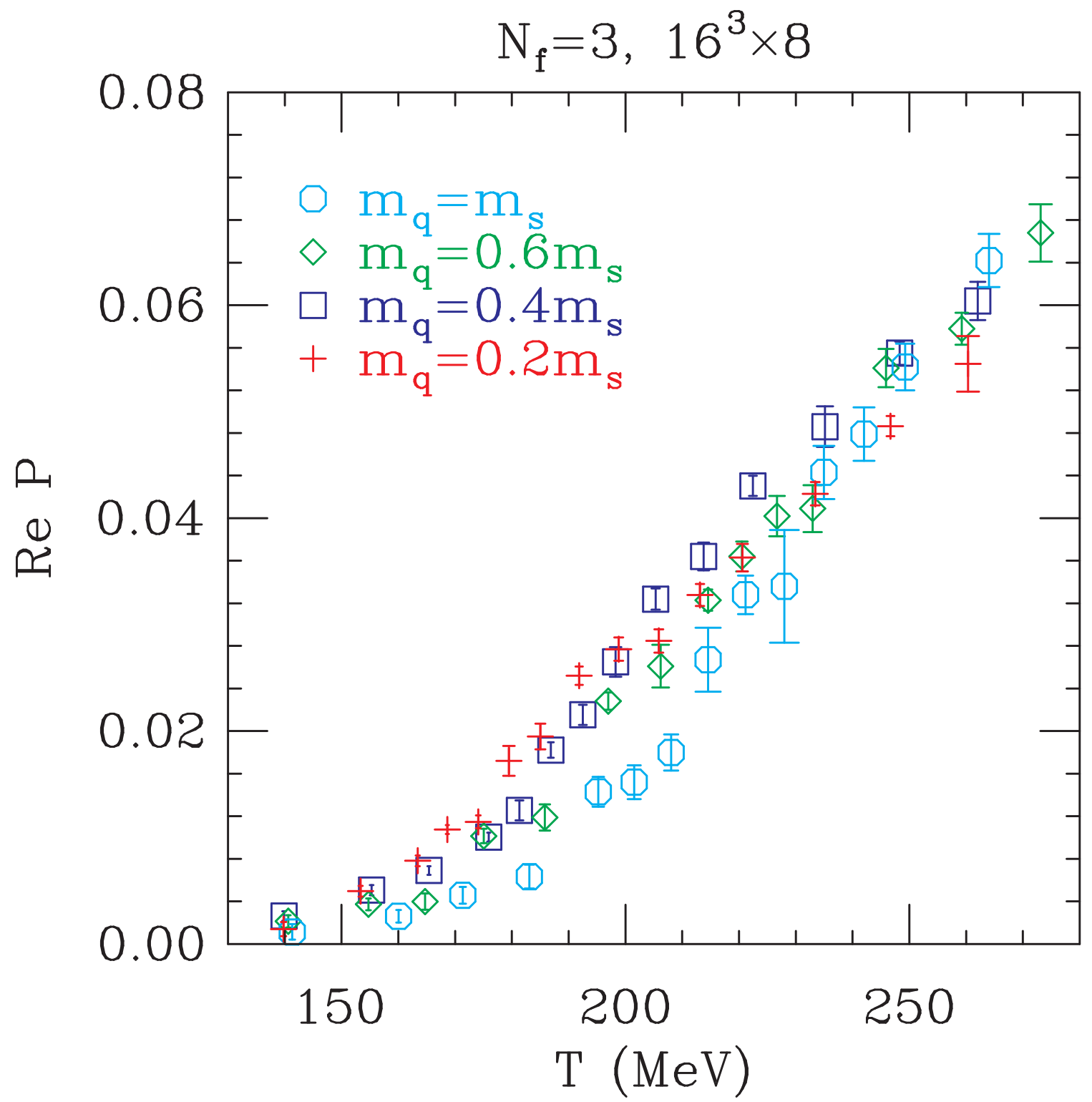

FIG. 2: The real part of the Polyakov loop as a function of temperature on $16^{3} \times 8$ lattices for three degenerate flavors of quarks with masses $m_{q} / m_{s}=1.0,0.6,0.4$ and 0.2 . 


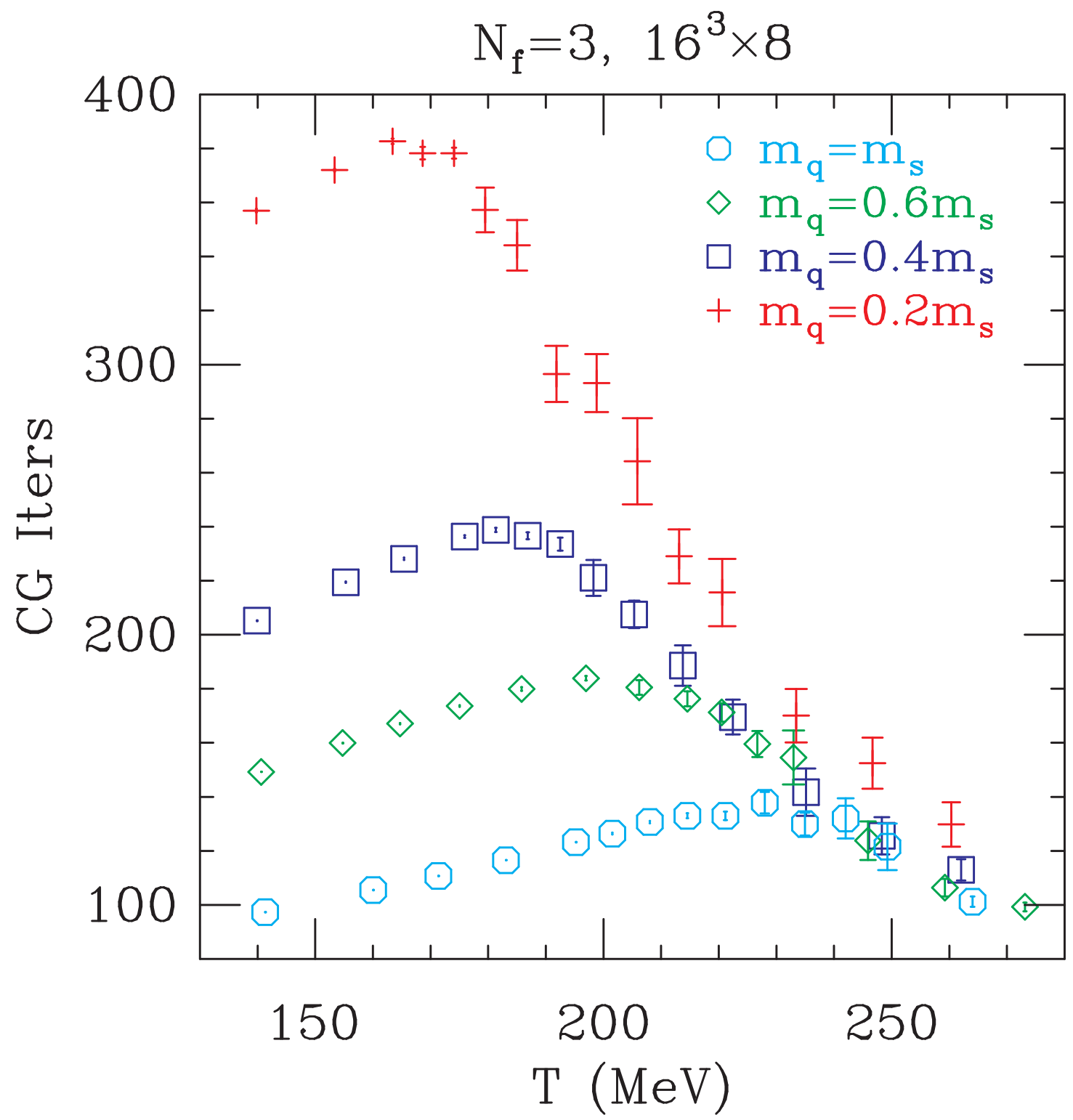

FIG. 3: The number of conjugate gradient iterations required for convergence of the inversion of the Dirac operator for three degenerate flavors of quarks with masses $m_{q} / m_{s}=1.0,0.6,0.4$ and 0.2 on $16^{3} \times 8$ lattices. 


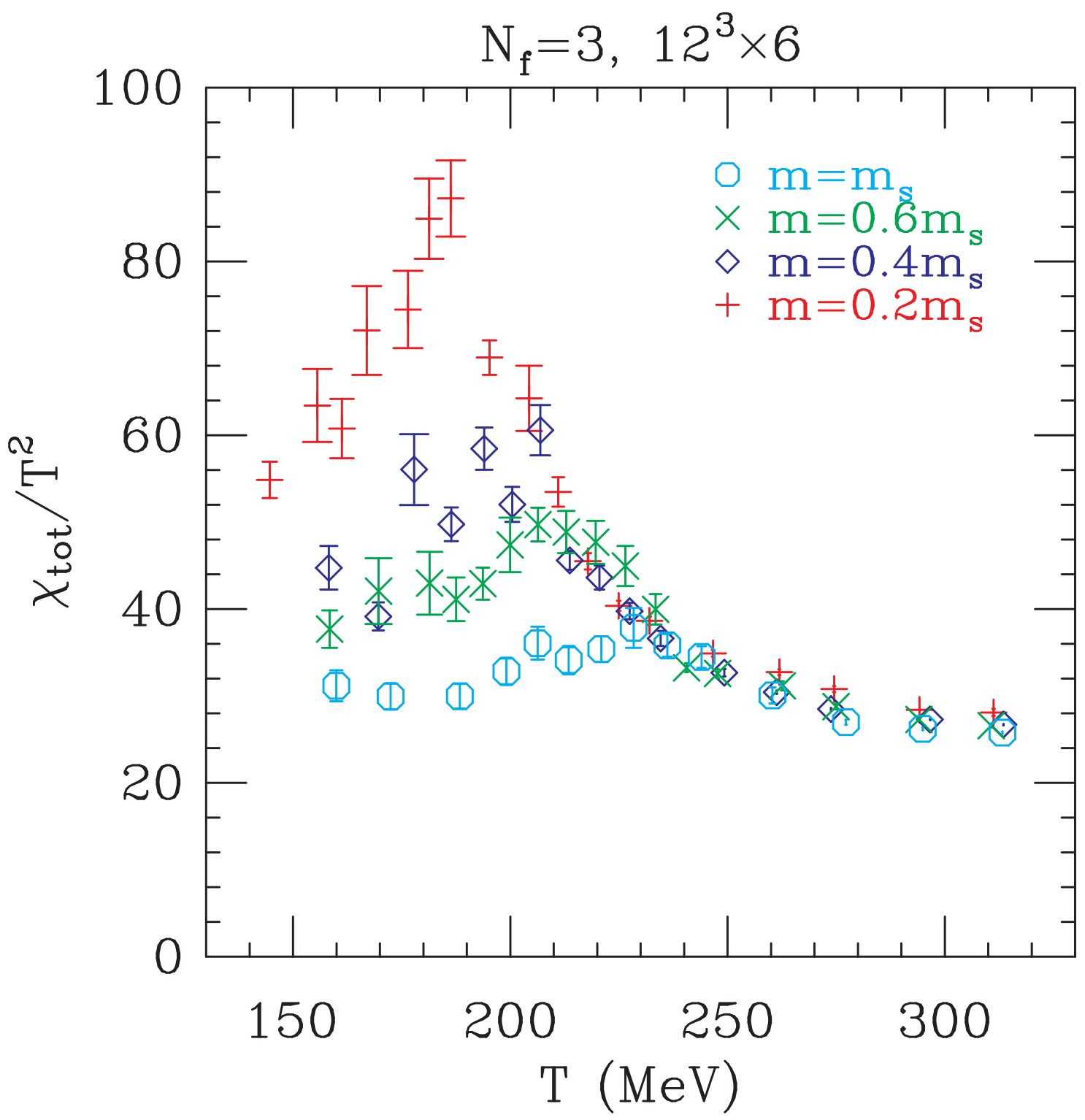

FIG. 4: The $\bar{\psi} \psi$ susceptibility as a function of temperature for three equal mass quarks on $12^{3} \times 6$ lattices. Results are shown for quark masses $m_{q} / m_{s}=1.0,0.6,0.4$, and 0.2 . Note the increase in the height of the peak as the quark mass is decreased. 


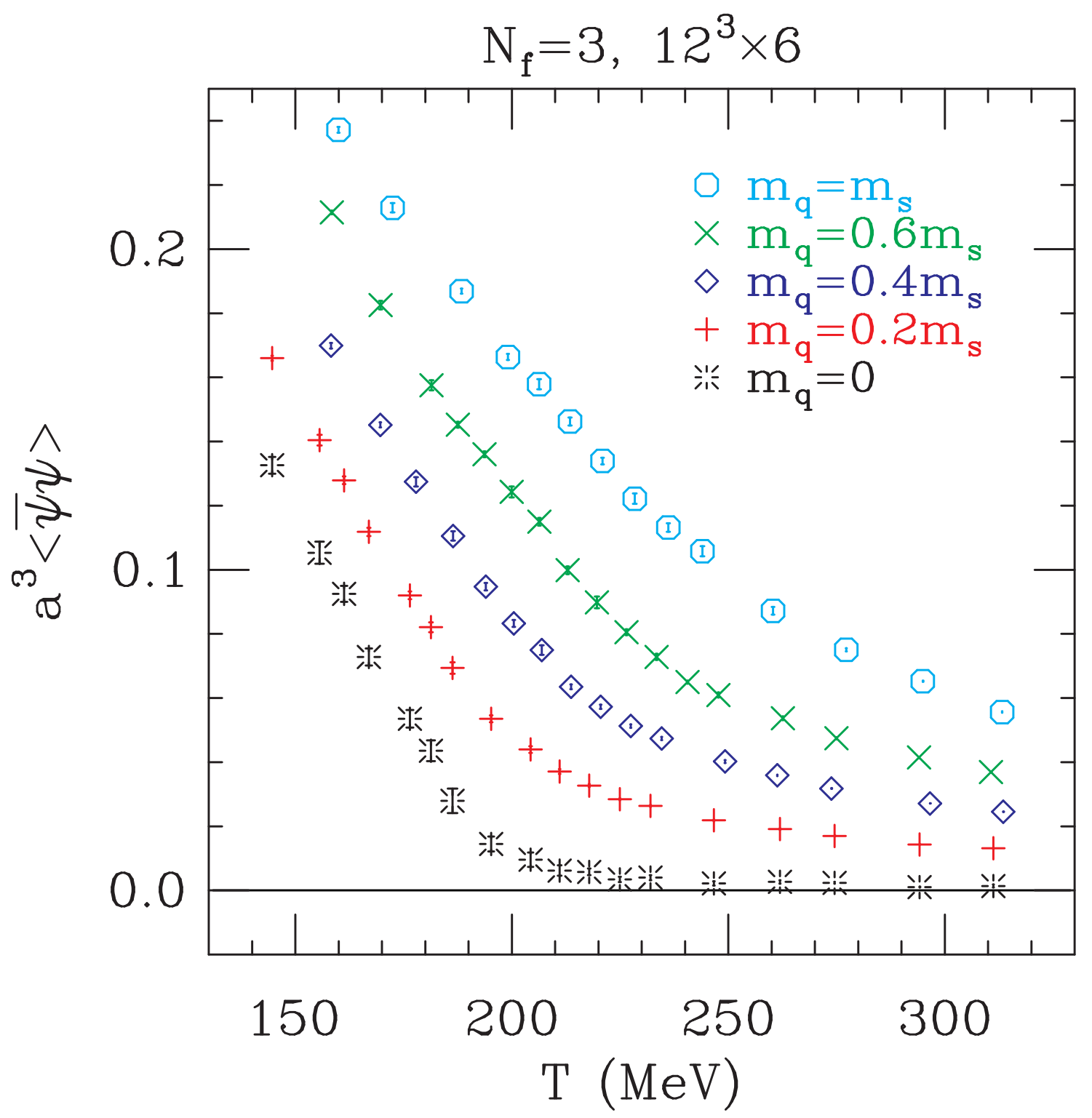

FIG. 5: The chiral order parameter, $\langle\bar{\psi} \psi\rangle$, as a function of temperature on $12^{3} \times 6$ lattices for $N_{f}=3$. The bursts are linear extrapolations of $\langle\bar{\psi} \psi\rangle$ for the two lowest quark masses to $m_{q}=0$ at fixed temperature. 


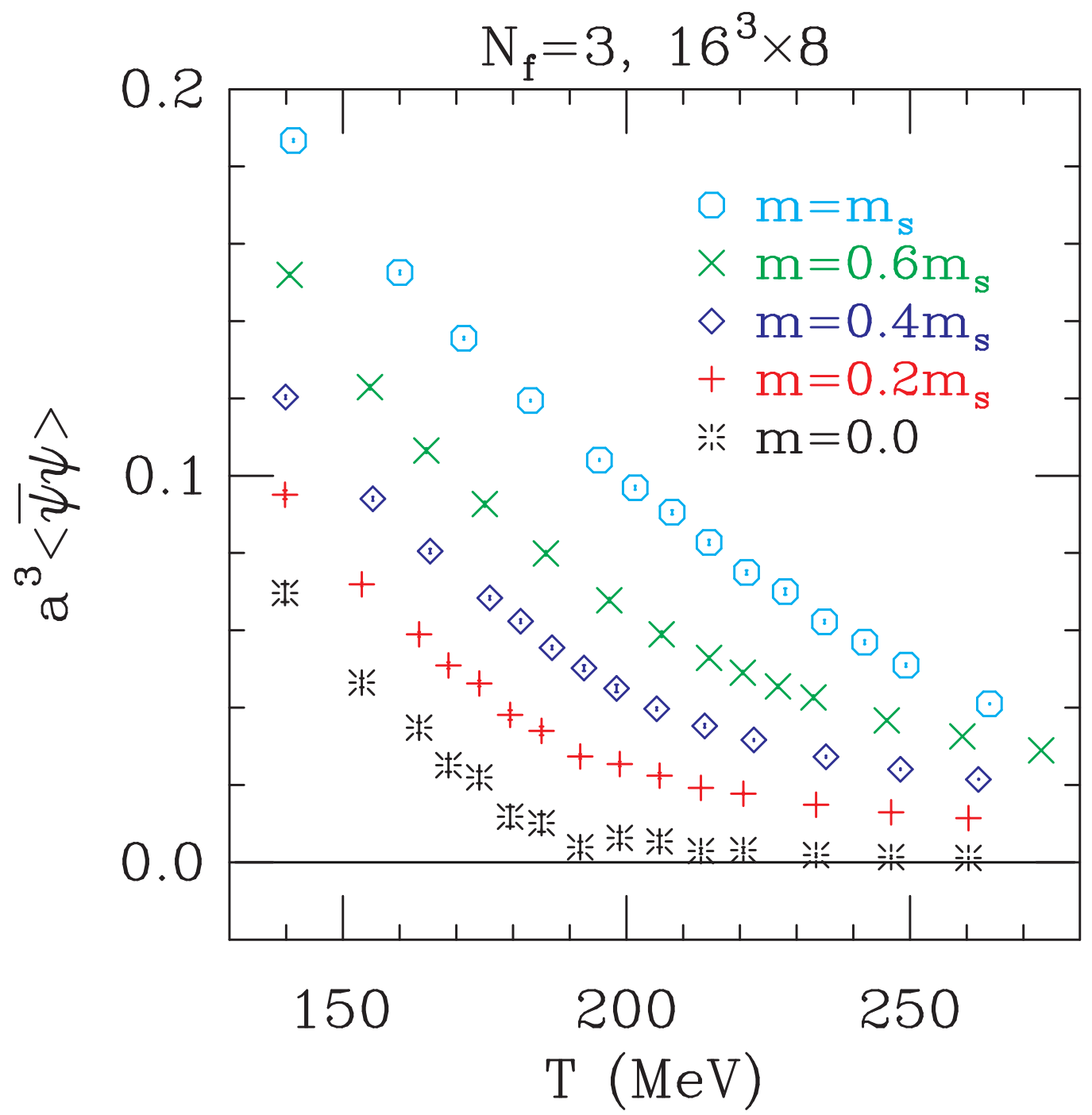

FIG. 6: The chiral order parameter $\langle\bar{\psi} \psi\rangle$ as a function of temperature on $16^{3} \times 8$ lattices for $N_{f}=3$. The bursts are linear extrapolations of $\langle\bar{\psi} \psi\rangle$ for the two lowest quark masses to $m_{q}=0$ at fixed temperature. 


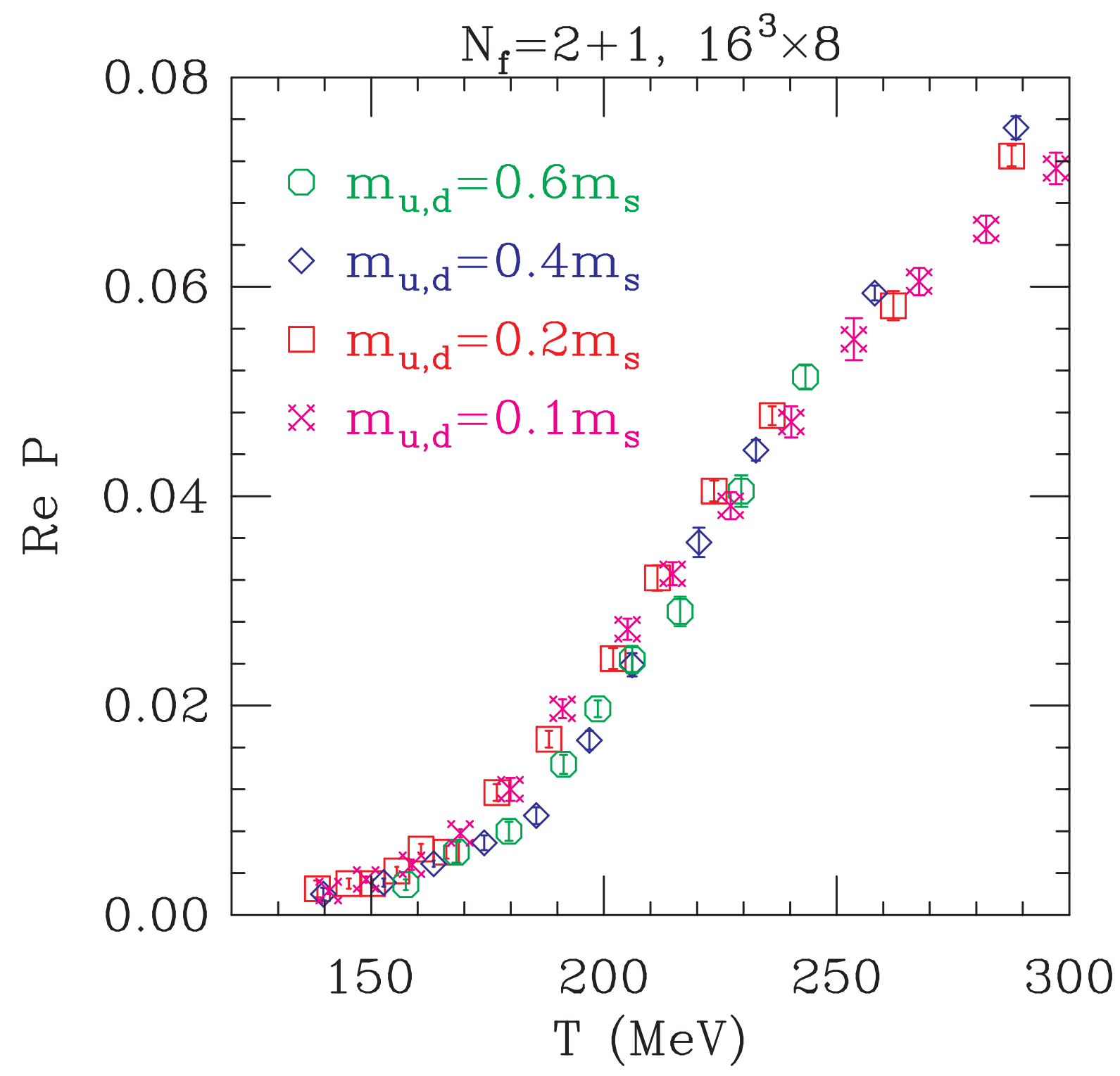

FIG. 7: The real part of the Polyakov loop as a function of temperature for two light and one heavy quark on $16^{3} \times 8$ lattices. Results are shown for light quark masses $m_{u, d} / m_{s}=0.6,0.4,0.2$ and 0.1. The mass of the heavy quark is fixed at $m_{s}$. 


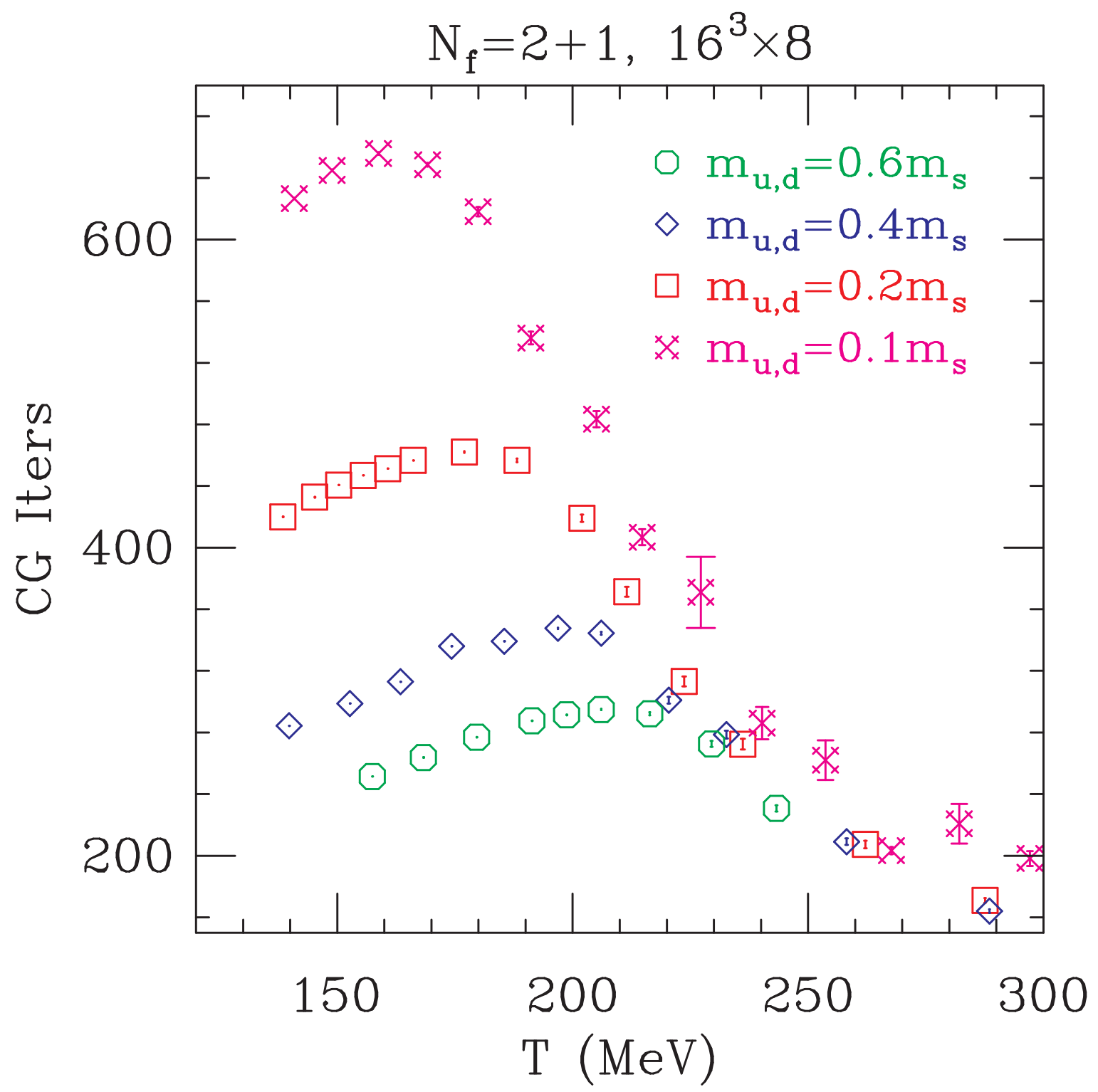

FIG. 8: The number of conjugate gradient iterations required for convergence of the inversion of the Dirac operator of light quarks on $16^{3} \times 8$ lattices. Results are shown for light quark masses $m_{u, d} / m_{s}=, 0.4,0.2$ and 0.1 . The mass of the heavy quark is fixed at $m_{s}$. 


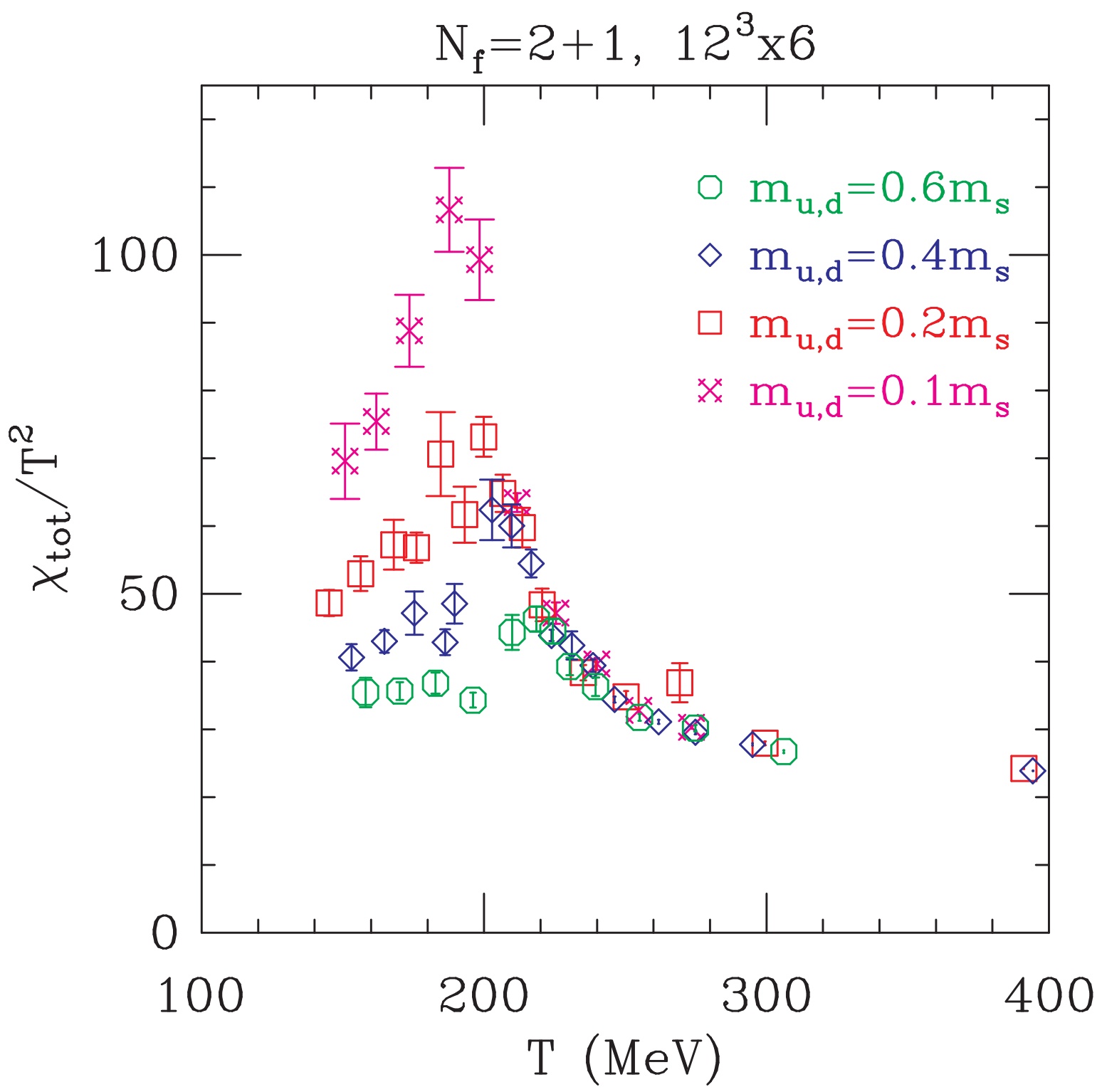

FIG. 9: The $\bar{\psi} \psi$ susceptibility as a function of temperature for two light and one heavy quark on $12^{3} \times 6$ lattices. Results are shown for light quark masses $m_{u, d} / m_{s}=0.6,0.4,0.2$ and 0.1 . The mass of the heavy quark is fixed at $m_{s}$. 


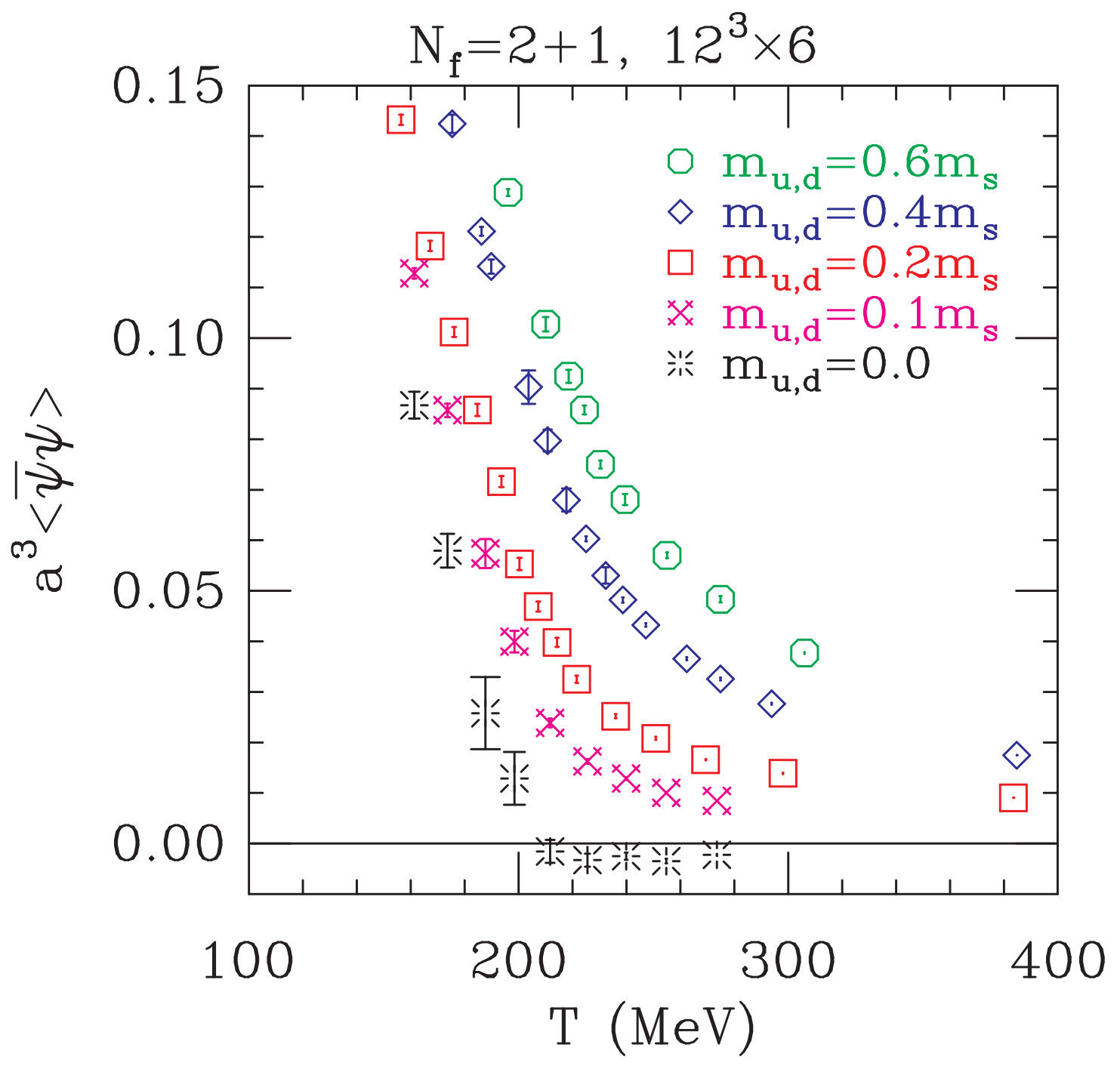

FIG. 10: The chiral order parameter $\bar{\psi} \psi$ as a function of temperature on $12^{3} \times 6$ lattices. The bursts are linear extrapolations in the quark mass to $m_{u d}=0$ for fixed temperature. 


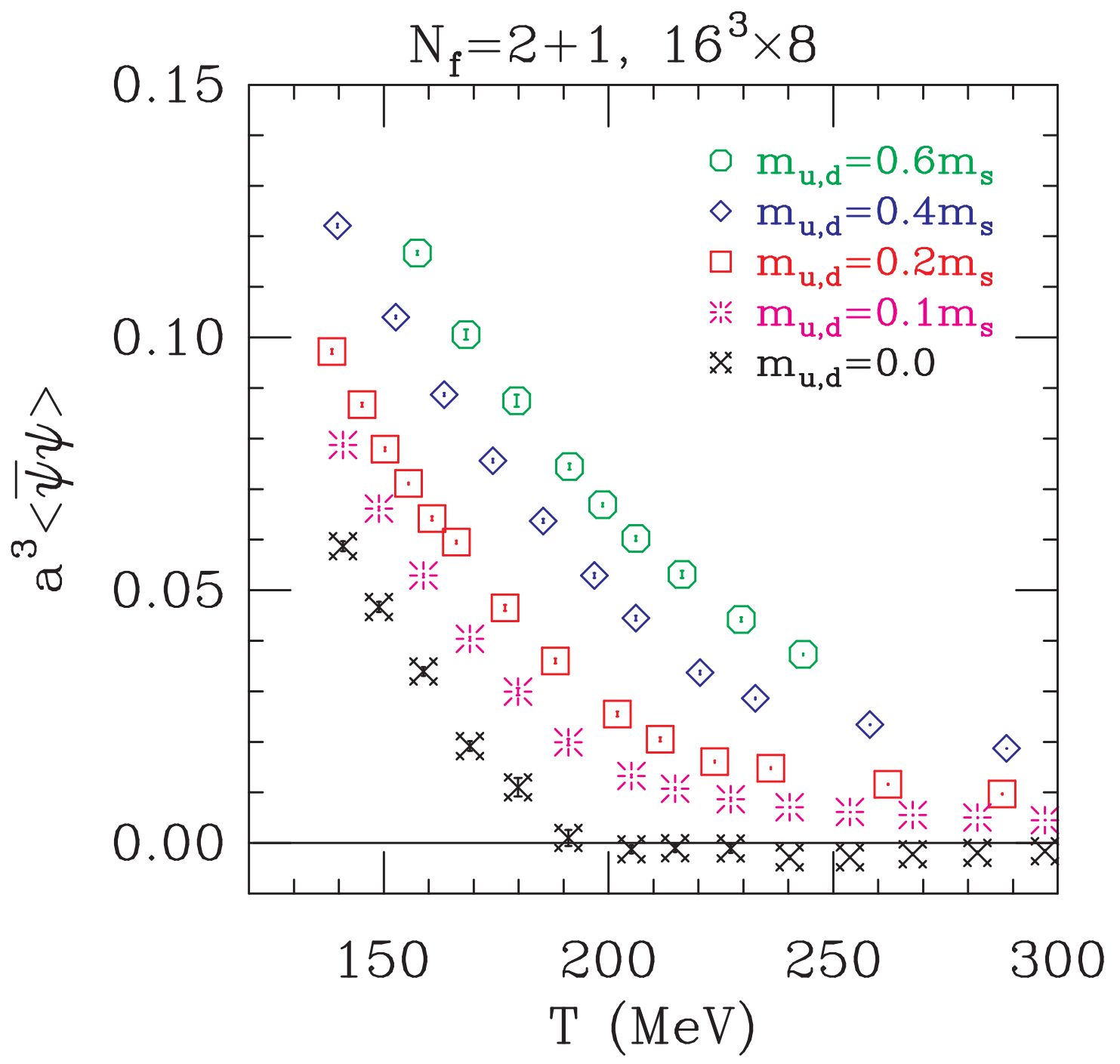

FIG. 11: The chiral order parameter $\bar{\psi} \psi$ on $16^{3} \times 8$ lattices. The bursts are linear extrapolations in the quark mass to $m_{u d}=0$ for fixed temperature. 


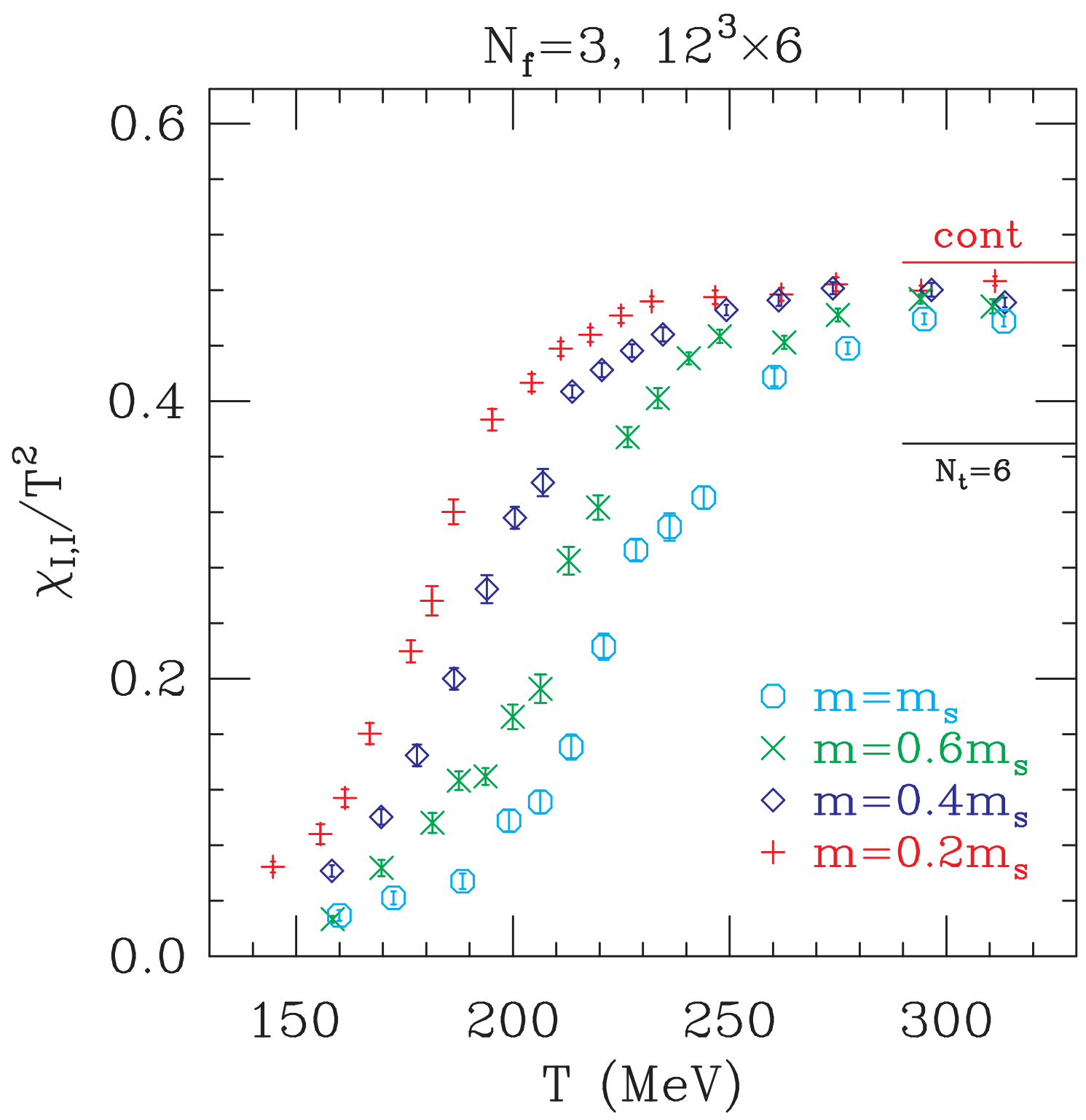

FIG. 12: The z-component of isospin susceptibility $\chi_{I, I}$ as a function of temperature for three degenerate flavors of quarks with masses $m_{q} / m_{s}=1.0,0.6,0.4$, and 0.2 on $12^{3} \times 6$ lattices. The solid lines on the right of the figure indicate the free quark value in the continuum and on a $12^{3} \times 6$ lattice. 


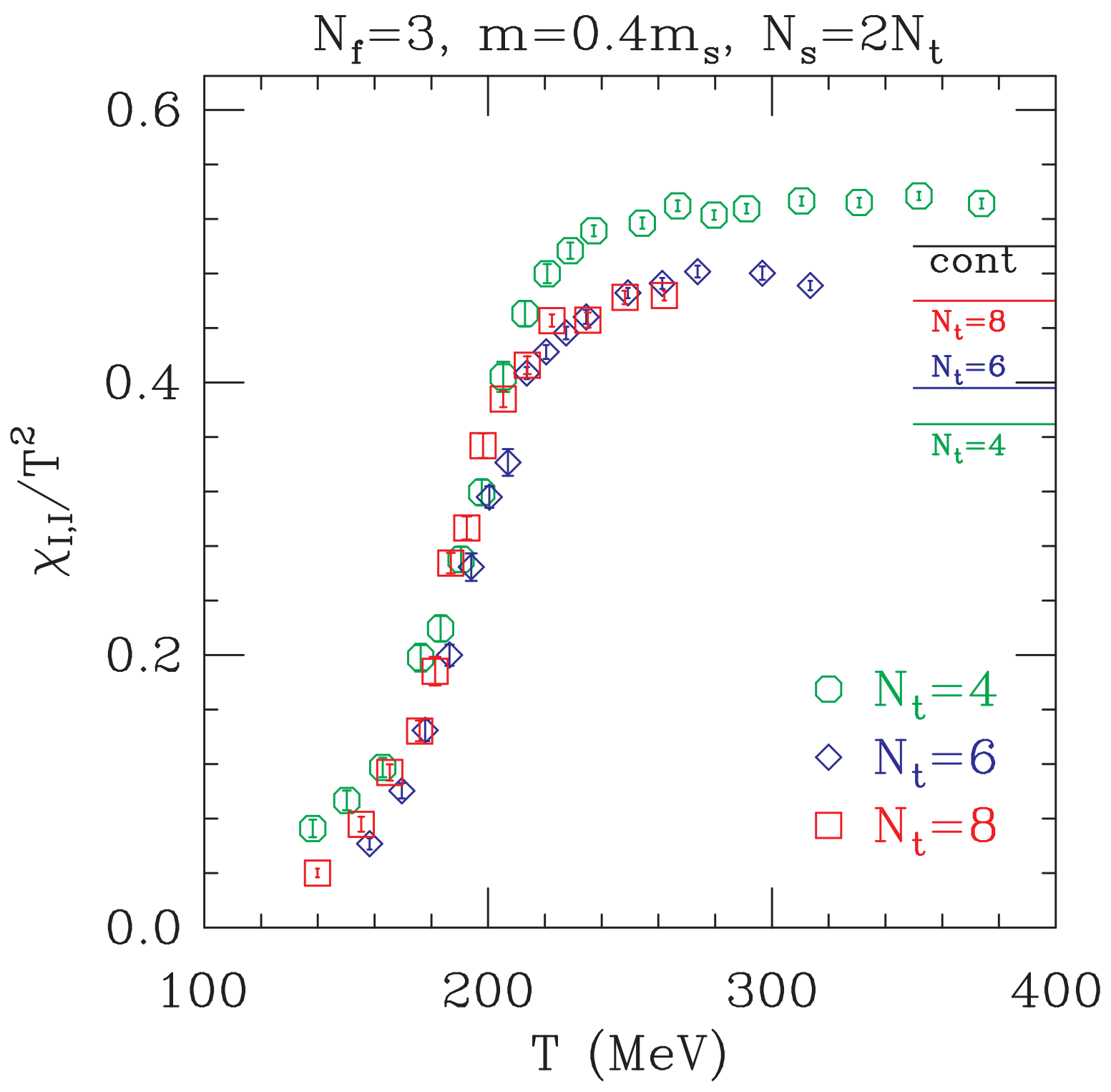

FIG. 13: The z-component of isospin susceptibility $\chi_{I, I}$ for three degenerate flavors of quarks with mass $m_{q}=0.4 m_{s}$, on $8^{3} \times 4,12^{3} \times 6$ and $16^{3} \times 8$ lattices. The solid lines on the right of the figure indicate the value for free quarks in the continuum, and on the finite lattices on which the simulations were carried out. The close agreement among these results is another indication of the excellent scaling properties of the Asqtad action. 


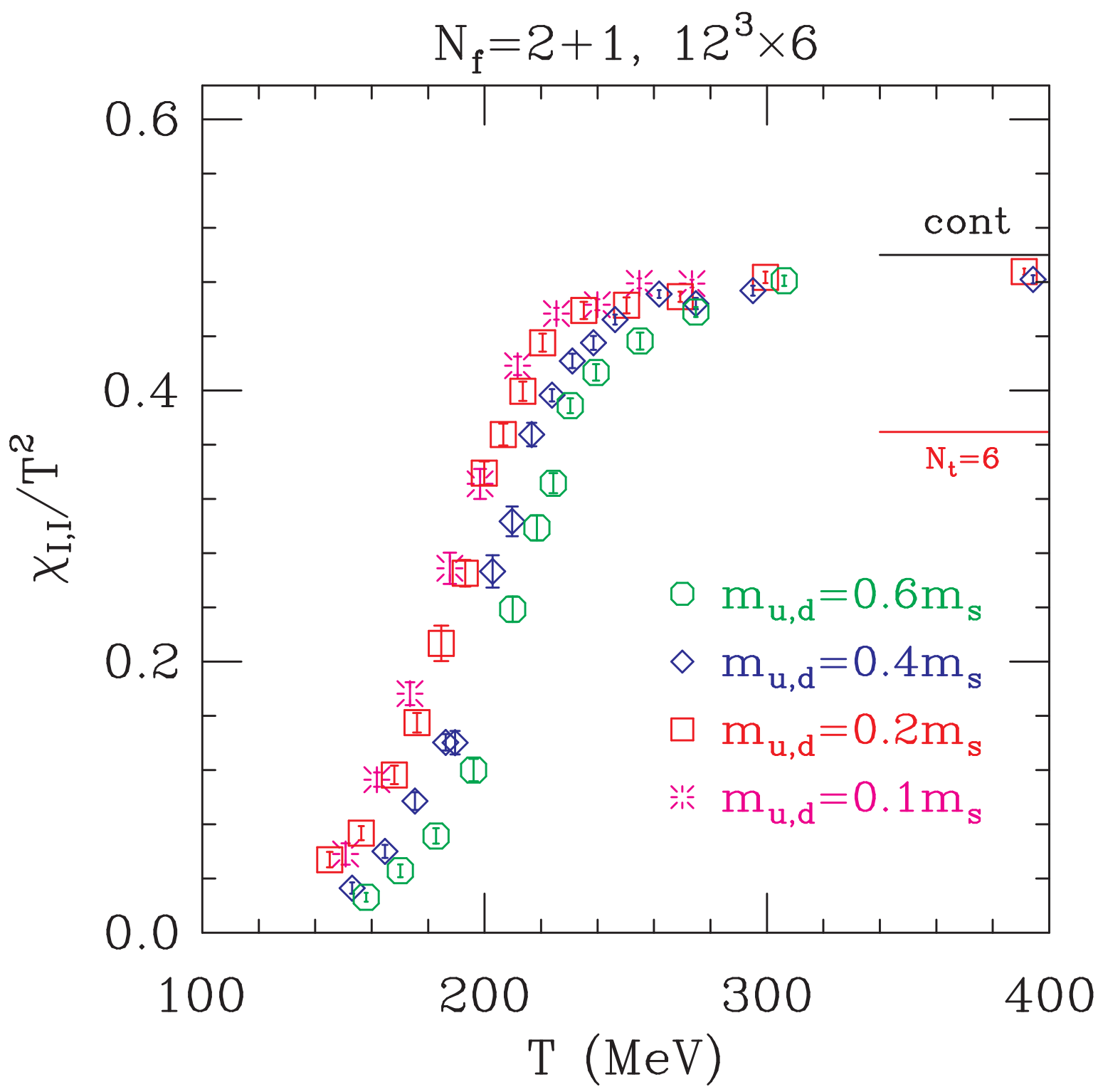

FIG. 14: The z-component of isospin susceptibility as a function of temperature for two light and one heavy quark on $12^{3} \times 6$ lattices. Results are shown for light quark masses $m_{u, d} / m_{s}=0.6,0.4$, 0.2 and 0.1 . The mass of the heavy quark is fixed at $m_{s}$. The solid lines on the right of the figure indicate the free quark value in the continuum and on a $12^{3} \times 6$ lattice. 


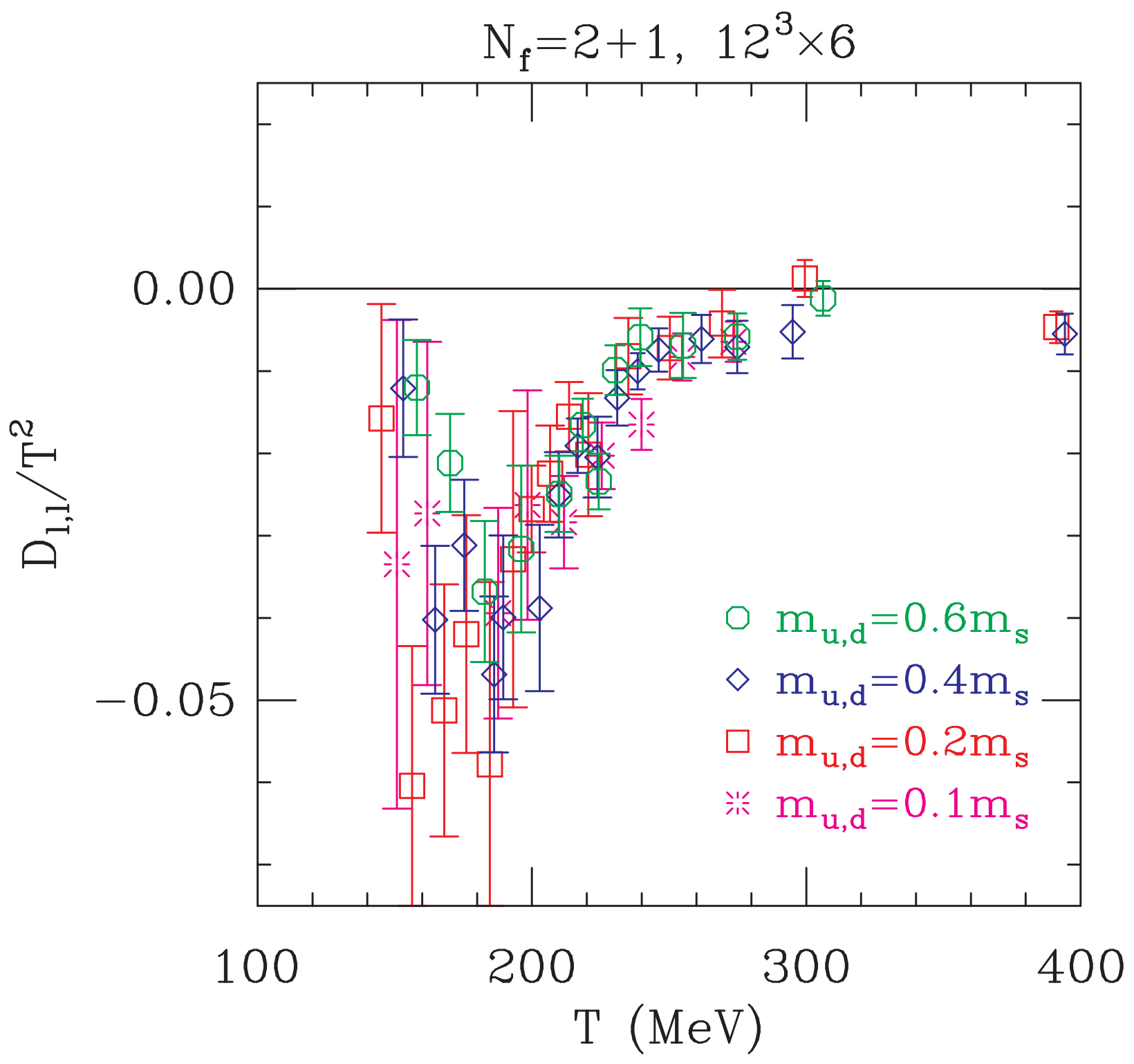

FIG. 15: The disconnected part of the light quark susceptibility $D_{l, l}$ as a function of temperature for two light and one heavy quark on $12^{3} \times 6$ lattices. Results are shown for light quark masses $m_{u, d} / m_{s}=0.6,0.4,0.2$ and 0.1 . The mass of the heavy quark is fixed at $m_{s}$. 


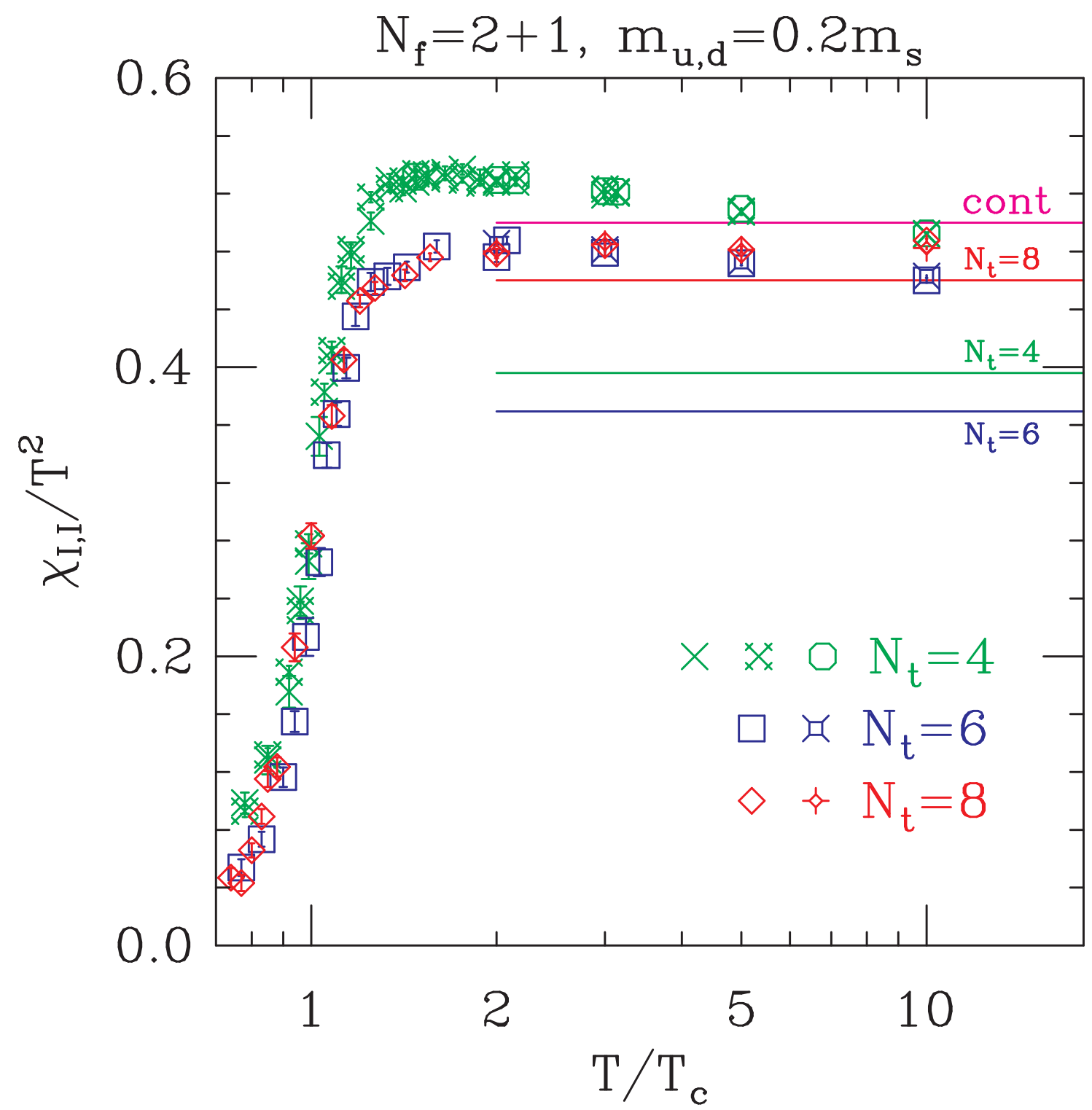

FIG. 16: The z-component of isospin susceptibilities as a function of $T / T_{c}$ for two light quarks with mass $0.2 m_{s}$ and one heavy quark with mass $m_{s}$. Results are shown for lattices with 4, 6 and 8 time slices. For $N_{t}=4$ the octagons, fancy crosses, and crosses are data from spatial volumes $16^{4}, 12^{3}$ and $8^{3}$, respectively. For $N_{t}=6$ the fancy squares and squares are data from spatial volumes $18^{3}$ and $12^{3}$, while for $N_{t}=8$ the fancy diamonds and diamonds are data from spatial volumes $24^{3}$ and $16^{3}$. The solid lines on the right of the figure indicate the value for free quarks in the continuum and on the finite lattices on which the simulations were carried out. The agreement between the $N_{t}=6$ and 8 results illustrate the excellent scaling property of the Asqtad action, and indicates that these results are close to the continuum ones. 


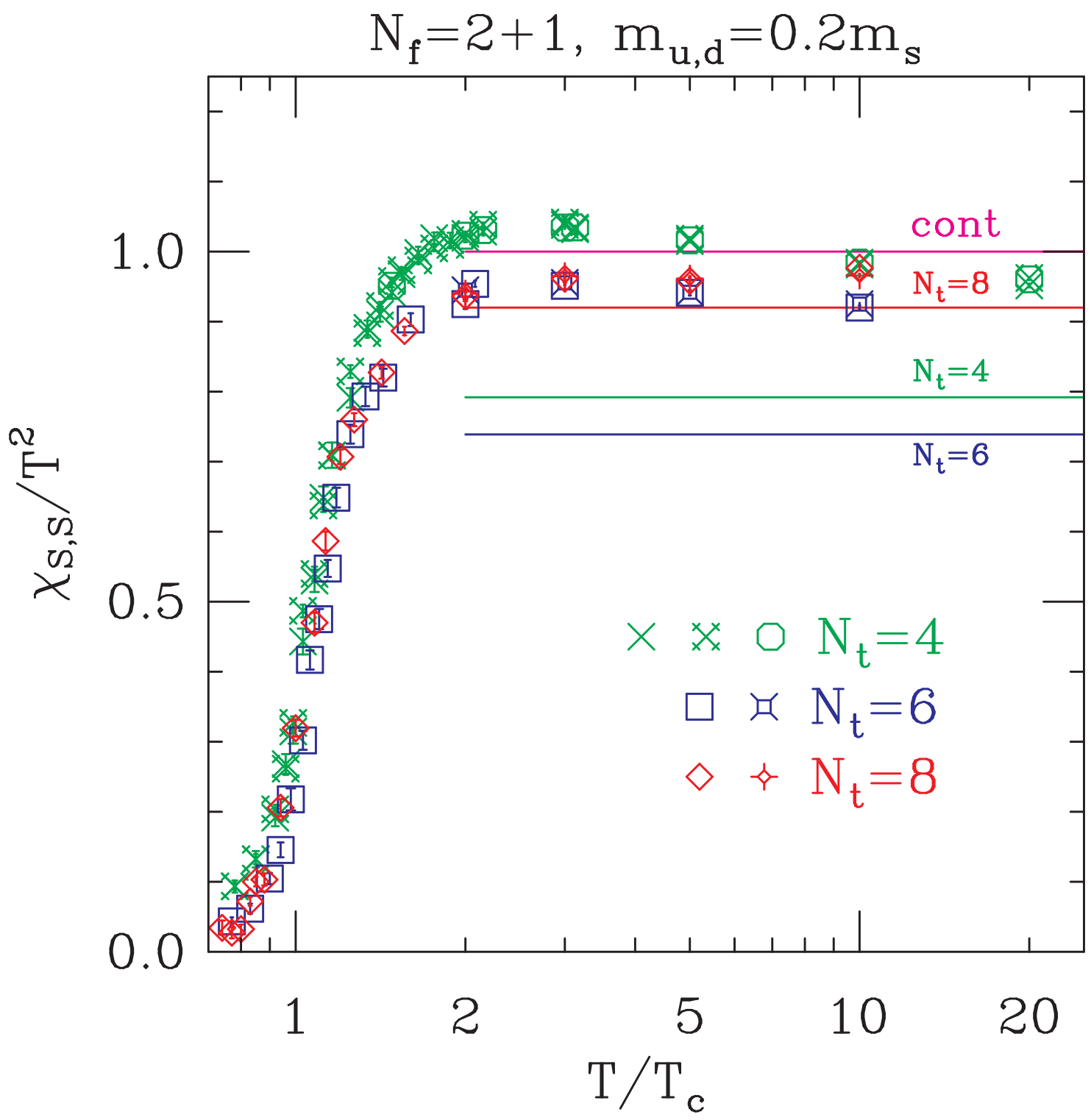

FIG. 17: The strange quark number susceptibility $\chi_{S, S}$ as a function of $T / T_{c}$ for two light quarks with mass $0.2 m_{s}$ and one heavy quark with mass $m_{s}$. Results are shown for lattices with 4, 6 and 8 times slices. The symbols have the same significance as in Fig. 16, The solid lines on the right of the figure indicate the value for free quarks in the continuum and on the finite lattices on which the simulations were carried out. 


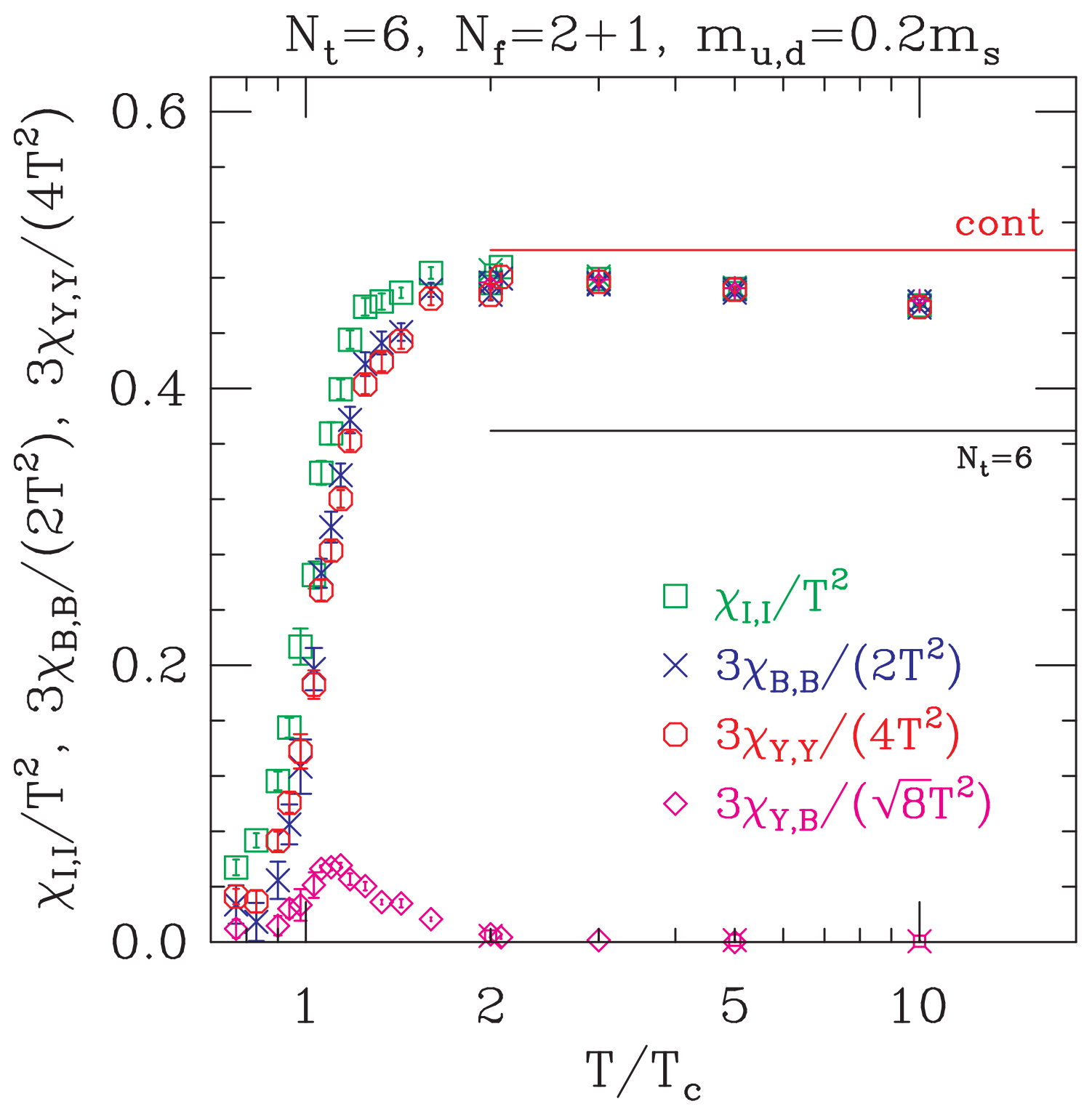

FIG. 18: The diagonal elements of the susceptibility matrix, $\chi_{I, I}, \chi_{B, B}$ and $\chi_{Y, Y}$ as a function of $T / T_{c}$ for two light quarks with mass $0.2 m_{s}$ and one heavy quark with mass $m_{s}$ on $12^{3} \times 6$ lattices. $\chi_{Y, Y}$ and $\chi_{B, B}$ have been normalized so that they approach the same limit as $\chi_{I, I}$ at high temperatures. Also shown is the off diagonal matrix element $\chi_{Y, B}$, which measures correlations between fluctuations in the hypercharge and baryon number. The coefficient of $\chi_{Y, B}$ is the geometric mean of those for $\chi_{Y, Y}$ and $\chi_{B, B}$. 